\title{
Dimensional analysis in relativity and in differential geometry
}

\author{
P.G.L. Porta Mana $\odot$ \\ Western Norway University of Applied Sciences, Bergen <pgleportamana.org>
}

13 December 2019; updated 20 May 2021

This note provides a short guide to dimensional analysis in Lorentzian and general relativity and in differential geometry. It tries to revive Dorgelo and Schouten's notion of 'intrinsic' or 'absolute' dimension of a tensorial quantity. The intrinsic dimension is independent of the dimensions of the coordinates and expresses the physical and operational meaning of a tensor. The dimensional analysis of several important tensors and tensor operations is summarized. In particular it is shown that the components of a tensor need not have all the same dimension, and that the Riemann (once contravariant and thrice covariant), Ricci (twice covariant), and Einstein (twice covariant) curvature tensors are dimensionless. The relation between dimension and operational meaning for the metric and stress-energy-momentum tensors is discussed; and the possible conventions for the dimensions of these two tensors and of Einstein's constant $\kappa$, including the curious possibility $\kappa=8 \pi G$ without $c$ factors, are reviewed.

per la piccola Emma

\section{Introduction}

From the point of view of dimensional analysis, do all components of a tensor need to have the same dimension? What happens to these components if we choose coordinates that don't all have dimensions of length or time? And if the components of a tensor have different dimensions, then does it make sense to speak of "the dimension of the tensor"? What are the dimensions of the metric and of the curvature tensors? What is the dimension of the constant in the Einstein equations?

A sense of insecurity gets hold of many students (and possibly of some researchers) in relativity, when they have to discuss and answer this kind of questions. This is evident in many question \& answer websites and wiki pages, where several incorrect or unfounded statements about dimensional analysis in relativity are in circulation. (For the record, the answers to the questions above are: 1 . No, they don't. 2 . See 
eq. (12) below. 3. Yes. 4. They are dimensionless. 5. $\mathrm{M}^{-1} \mathrm{~L}^{-1} \mathrm{~T}^{2}$, or $\mathrm{M}^{-1} \mathrm{~L}$, or $M^{-1} L^{3} T^{-2}$, depending on the dimensions you assign to the metric and stress-energy-momentum tensors, see $\S \S 9-11$. )

Several factors contribute to these misconceptions and insecurity. Modern texts in Lorentzian and general relativity commonly use geometrized units. They say that, for finding the dimension of some constant in a tensorial equation, it's sufficient to compare the dimensions of the terms in the equation. But the application of this procedure is sometimes not so immediate, because some tensors don't have universally agreed dimensions - prime example the metric tensor. Many texts use four coordinates with dimension of length, and base their dimensional analyses on that specific choice ${ }^{1}$, multiplying timelike tensorial components by appropriate powers of $c$. Such common practices can therefore give students the impression that coordinates ought to always be lengths, and that all components of a tensor ought to have the same dimension. Yet, students cannot find such rules explicitly stated anywhere. We'll see shortly that no such rules in fact exist, nor are they necessary.

Dimensional analysis is thus not very self-evident in relativity and in differential geometry. The present note wants to provide a short but exhaustive guide to it. Some important dimensional-analysis questions in general relativity are also consistently settled here; for example the dimension of the Riemann curvature tensor, or the effect of the covariant or Lie derivatives on dimensions.

The application of dimensional analysis in relativity is most straightforward and self-evident if we rely on the coordinate-free or intrinsic approach to differential geometry, briefly recalled below, and if we adopt the perhaps overlooked notion of intrinsic dimension of a tensor. The intrinsic dimension of a tensor was introduced under the name 'absolute dimension' by Schouten and Dorgelo ${ }^{2}$ and used in Truesdell \& Toupin ${ }^{3}$ and recently in Hehl \& Obukhov ${ }^{4}$. As its name implies, this dimension is independent of the choice and dimensions of coordinate functions. It is distinct from the dimensions of the tensor's components, which instead depend on the dimensions of the coordinates. The intrinsic dimension of

1 e.g. Tolman 1949 p. 71 eq. (37.1); Landau \& Lifshitz 1996 p. 80 eq. (32.15); for exceptions with coordinates with mixed dimensions see e.g. Fock $1964 \S$ V.55; McVittie 1965 § 4.1; Fokker 1965 § VII.1; Truesdell \& Toupin 1960 § F.III.280; Kitano 2013 § X.

2 Dorgelo \& Schouten 1946; Schouten 1989 ch. VI. Appendix II. $\quad{ }^{\mathbf{4}}$ Hehl \& Obukhov 2003 § B.1; 2005a.

3 Truesdell \& Toupin 1960 
a tensor is determined by the latter's physical and operational ${ }^{5}$ meaning. It is therefore a natural notion for dimensional analysis in relativity.

Here is a synopsis of the rest of this note. The intrinsic approach to differential geometry is outlined, with references, in the next section, together with some notation necessary to our discussion. Section 3 gives a simple example of dimensional analysis for a two-dimensional spacetime. This example might be enough for most readers to grasp the basic way of reasoning; such readers can work out the rest for themselves whenever they need and don't need to read the rest of this note. Sections 4-7 offer a more systematic discussion and a synopsis of dimensional analysis for the main tensorial operations. The notion of intrinsic dimension is explained in $\S 5$. The intrinsic dimensions of various curvature tensors, of the metric tensor, and of the stress-energy-momentum tensor are discussed in $\S \S 8-10$. In particular, the contravariant and thrice covariant Riemann, twice covariant Ricci, and twice covariant Einstein curvature tensors are found to have intrinsic dimension 1 , that is, to be dimensionless. The operational motivation of several standard choices for the dimensions of the metric and stress-energy-momentum tensors are also discussed. The possible dimensions of the constant in the Einstein equations are finally derived in $\S 11$.

This note obviously assumes familiarity with basic tensor calculus and related notions, for example of co- and contra-variance, tensor product, contraction. Some passages assume familiarity with the exterior calculus of differential forms. The general ideas, however, should be understandable even without such familiarity.

Finally, quoting Truesdell \& Toupin ${ }^{6}$, "dimensional analysis remains a controversial and somewhat obscure subject. We do not attempt a complete presentation here". References about recent developments in this subject are given in the summary of $\S 12$.

\section{Intrinsic view of differential-geometric objects: brief reminder and notation}

From the intrinsic point of view, a tensor is defined by its geometric properties. For example, a vector field $v$ is an object that operates on functions defined on the (spacetime) manifold, yielding new functions,

\footnotetext{
${ }^{5}$ Bridgman 1958. $\quad \mathbf{6}^{\mathbf{6}}$ Truesdell \& Toupin 1960 Appendix $\S 7$ footnote 4.
} 
with the properties $v(a f+b g)=a v(f)+b v(g)$ and $v(f g)=v(f) g+f v(g)$ for all functions $f, g$ and reals $a, b$. A covector field (also called 1-form) $\boldsymbol{\omega}$ is an object that operates on vector fields, yielding functions, with the property $\boldsymbol{\omega}(f \boldsymbol{u}+g \boldsymbol{v})=f \boldsymbol{\omega}(\boldsymbol{u})+g \boldsymbol{\omega}(\boldsymbol{v})$ for all vector fields $\boldsymbol{u}, \boldsymbol{v}$ and functions $f, g$. The sum of vector or covector fields and their products by functions are defined in an obvious way. Tensors are constructed from these objects; see also the end of this section for a slightly different point of view.

A system of coordinates $\left(x^{i}\right)$ is just a set of linearly independent functions. This set gives rise to a set of vectors fields $\left(\frac{\partial}{\partial x^{i}}\right)$ and to a set of covector fields $\left(\mathrm{d} x^{i}\right)$ by the obvious requirements that $\frac{\partial}{\partial x^{i}}\left(x^{j}\right)=\delta_{i}^{j}$ and $\mathrm{d} x^{i}\left(\frac{\partial}{\partial x^{j}}\right)=\delta^{i}{ }_{j}$. These two sets can be used as bases to express all other vectors and covectors as linear combinations. A vector field $v$ can thus be written as

$$
v \equiv \sum_{i} v^{i} \frac{\partial}{\partial x^{i}} \equiv v^{i} \frac{\partial}{\partial x^{i}}
$$

where the functions $v^{i}:=\mathrm{d} x^{i}(v)$ are its components with respect to the basis $\left(\frac{\partial}{\partial x^{i}}\right)$. Analogously for a covector field.

For the presentation of the intrinsic view I recommend the excellent texts by Choquet-Bruhat et al. (1996), Boothby (2003), Abraham et al. (1988), Burke (1987), Bossavit (1991), and more on the general-relativity side Misner et al. (1973 ch. 9), Gourgoulhon (2012 ch. 2), Penrose \& Rindler (2003).

For the notation in dimensional analysis I use Iso conventions: ${ }^{7}$ $\operatorname{dim}(\boldsymbol{A})$ is the dimension of the quantity $\boldsymbol{A}$, and among the base quantities are mass $M$, length $L$, time $T$, temperature $\Theta$, electric current I, and the dimensionless 1 . Note that I don't discuss units - it doesn't matter here whether the unit for length is the metre or the furlong, for example.

Throughout this note $c$ denotes the speed of light, with $\operatorname{dim}(c)=\mathrm{LT}^{-1}$. Its numerical quantity value $\{c\}$ depends on the chosen units of length and time.

The number, ordering, and symmetries of a tensor's covariant and contravariant "slots" ${ }^{8}$ will be important in our discussion. The traditional coordinate-free notation ' $\boldsymbol{A}$ ' omits this information. We thus need a coordinate-free notation that makes it explicit when needed. Penrose \&

\footnotetext{
${ }^{7}$ IsO $2009 \S 5 . \quad{ }^{8}$ Misner et al. $1973 \S 3.2$.
} 
Rindler ${ }^{9}$ propose an abstract-index notation where ' $A_{i}^{j k}$, , for example, denotes a tensor covariant in its first slot and contravariant in its second and third slots. Every index in this notation is "a label whose sole purpose is to keep track of the type of tensor under discussion"10. So this notation doesn't stand for a component of the tensor. For the latter, Penrose \& Rindler use bold indices instead: ' $A_{i}^{j k}$, . But in our discussion the difference between a tensor and its set of components is crucial, and Penrose \& Rindler's abstract-index notation unfortunately lends itself to conceptual and typographic misunderstanding.

I shall therefore use a notation such as $\boldsymbol{A}_{\bullet} \cdot \boldsymbol{*}$ to indicate that $\boldsymbol{A}$ is covariant in its first slot and contravariant in its second and third slots. Its components would thus be $\left(A_{i}^{j k}\right)$. For brevity I'll call this a 'co-contracontra-variant' tensor, with an obvious naming generalization for other tensor types. A set of completely antisymmetric slots will be put within bars: thus the notation $\boldsymbol{A}_{|\cdot \bullet|}^{{ }_{|.0|}}$ means that $\boldsymbol{A}$ is completely antisymmetric in its last two covariant slots. Finally, in accord with convenient modern terminology, completely antisymmetric contravariant tensors of order $k$ will be called ' $k$-vectors'; and completely antisymmetric covariant tensors of order $k$, ' $k$-covectors'. The terms 'multi-vector' and 'multi-covector' are used when $k$ isn't specified.

The only weak points of the notation just explained are the operations of transposition and contraction, which the index notation depicts so well instead. Considering that transposition is a generalization of matrix transposition, and contraction a generalization of trace, I'll use the following notation:

- $\boldsymbol{A}^{\top \alpha \beta}$ is the transposition (swapping) of the $\alpha$ th and $\beta$ th slots. Its coordinate-free definition is

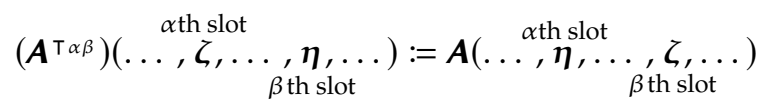

for all $\zeta, \boldsymbol{\eta}$ of appropriate variance type.

- $\operatorname{tr}_{\alpha \beta} \boldsymbol{A}$ is the contraction of the $\alpha$ th and $\beta$ th slots, which must have opposite variance types; note that we may have $\beta<\alpha$. Its coordinate-free

9 Penrose \& Rindler 2003 § 2.2. $\quad 10$ Penrose \& Rindler 2003 p. 75. 
definition is

$$
\left(\operatorname{tr}_{\alpha \beta} \boldsymbol{A}\right)(\ldots, \ldots, \ldots):=\sum_{i} \boldsymbol{A}\left(\ldots, \boldsymbol{u}_{i}, \ldots, \underset{\beta \text { th slot }}{\left.\boldsymbol{\omega}^{i}, \ldots\right)}\right.
$$

for any arbitrary complete and linearly independent sets $\left\{\boldsymbol{u}_{i}\right\},\left\{\boldsymbol{\omega}^{j}\right\}$ such that $\boldsymbol{\omega}^{j}\left(\boldsymbol{u}_{i}\right)=\delta^{j}{ }_{i}$.

In index notation the two operations above are the familiar

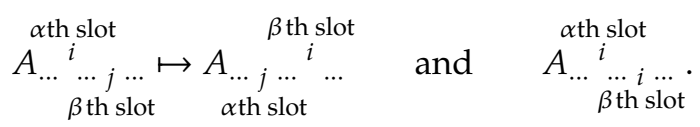

For the sake of notation economy I'll denote the contraction of adjacent slots of two tensors by simple juxtaposition. For example, if $\boldsymbol{A} \equiv \boldsymbol{A}_{\bullet}$, $\boldsymbol{B} \equiv \boldsymbol{B}_{\text {... }}$ and $v$ is a vector, then

$$
\boldsymbol{A B}:=\operatorname{tr}_{23}\left(\boldsymbol{A}_{\bullet}^{\bullet} \otimes \boldsymbol{B}_{\text {.. }}\right), \quad \boldsymbol{B} \boldsymbol{v}:=\operatorname{tr}_{23}\left(\boldsymbol{B}_{. .} \otimes \boldsymbol{v}^{\bullet}\right) .
$$

This notation makes sense considering tensors as linear operators.

Contraction and transposition will be discussed only sparsely, so I hope you won't find the notation above too uncomfortable.

It is possible to build the tensor-product architecture not on vectors and covectors, but on multi-vectors and multi-covectors, with their straight and twisted (also called 'even' and 'odd', or 'polar' and 'axial') orientations. This elegant and powerful geometric point of view leads to deeper physical insights and is gaining popularity in the literature. For its presentation I recommend the texts of Bossavit (1991 especially ch. 3), Burke (1983; 1987; 1980; 1995), de Rham (1984 ch. 2), Schouten (1954), Deschamps (1970; 1981), Lindell (2004).

In the notation above, the bars identify $k$-vectors and $k$-covectors for $k>1$. Thus $\boldsymbol{A}_{|\cdot .|}^{\cdot}$ indicates that $\boldsymbol{A}$ belongs to the tensor product of 1 -vectors and 2-covectors; it's also called a vector-valued 2-covector. To avoid burdening the notation I won't add symbols denoting straight or twisted orientation, but I'll explicitly state in the text when any object has a twisted orientation.

\section{An introductory two-dimensional example}

Let me first present a simple example of dimensional analysis for a two-dimensional spacetime. I provide very little explanation, letting the 
analysis speak for itself. The next sections will give a longer discussion of the general point of view, of the assumptions, and of cases with more elaborate geometric objects.

In a region of a two-dimensional spacetime we use coordinates $(x, y)$. These coordinates allow us to uniquely label every event in the region (otherwise they wouldn't be coordinates). Let us say that coordinate $x$ has dimension of temperature, and $y$ of specific entropy:

$$
\operatorname{dim}(x)=\Theta, \quad \operatorname{dim}(y)=\mathrm{s}:=\mathrm{L}^{2} \mathrm{~T}^{-2} \Theta^{-1} .
$$

This choice could be possible for several reasons. For example, the region could be occupied by a heat-conducting material; in a specific spacetime foliation, its temperature increases along each 1-dimensional spacelike slice, and its entropy density is uniform on each slice but increases from slice to slice. ${ }^{11}$ Owing to this kind of monotonic behaviour for these quantities, if we are given a pair of temperature \& specific-entropy values we can identify a unique event associated to them in this spacetime region. They can thus be used as a coordinate system. The point here is that coordinates can have any dimensions, because of physical reasons. In atmospheric and ocean dynamics, for example, pressure or mass density are sometimes used as coordinates for depth ${ }^{12}$.

From these coordinates we construct two covector fields $(\mathrm{d} x, \mathrm{~d} y)$, and two vector fields $\left(\frac{\partial}{\partial x}, \frac{\partial}{\partial y}\right)$ that serve as bases for the spaces of tangent covectors, vectors, and tensors. Their dimensions are

$$
\begin{aligned}
\operatorname{dim}(\mathrm{d} x) & =\Theta & \operatorname{dim}(\mathrm{d} y) & =\mathrm{s} \\
\operatorname{dim}\left(\frac{\partial}{\partial x}\right) & =\Theta^{-1} & \operatorname{dim}\left(\frac{\partial}{\partial y}\right) & =\mathrm{s}^{-1}
\end{aligned}
$$

Consider a contra-co-variant tensor field $\boldsymbol{A} \equiv \boldsymbol{A}^{\bullet}$. in this region. Using the basis fields above it can be written as

$$
\boldsymbol{A}=A^{x}{ }_{x} \frac{\partial}{\partial x} \otimes \mathrm{d} x+A^{x}{ }_{y} \frac{\partial}{\partial x} \otimes \mathrm{d} y+A^{y}{ }_{x} \frac{\partial}{\partial y} \otimes \mathrm{d} x+A^{y} y \frac{\partial}{\partial y} \otimes \mathrm{d} y,
$$

where $A_{x}^{x}:=\boldsymbol{A}\left(\mathrm{d} x, \frac{\partial}{\partial x}\right), A^{x}{ }_{y}:=\boldsymbol{A}\left(\mathrm{d} x, \frac{\partial}{\partial y}\right)$, and so on, are the components of the tensor in the coordinate system $(x, y)$.

11 For general-relativistic thermomechanics see e.g. Eckart 1940; Maugin 1974; 1978a,b,c,d; Muschik \& von Borzeszkowski 2014. $\quad 12$ Griffies 2004 ch. 6; Vallis $2006 \S 2.6 .2$. 
By the rules of dimensional analysis, the two sides of the expansion above, and in fact each summand on the right side, must have the same dimension. Denoting $A:=\operatorname{dim}(\boldsymbol{A})$, we thus find the four equations

$$
\mathrm{A}=\operatorname{dim}\left(A^{x}{ }_{x}\right)=\operatorname{dim}\left(A^{x}{ }_{y}\right) \Theta^{-1} \mathrm{~s}=\operatorname{dim}\left(A^{y}{ }_{x}\right) \Theta \mathrm{s}^{-1}=\operatorname{dim}\left(A^{y}{ }_{y}\right),
$$

or

$$
\begin{array}{ll}
\operatorname{dim}\left(A^{x}{ }_{x}\right)=\mathrm{A} & \operatorname{dim}\left(A^{x}{ }_{y}\right)=\mathrm{A} \Theta \mathrm{s}^{-1} \equiv \mathrm{AL}^{-2} \mathrm{~T}^{2} \Theta^{2} \\
\operatorname{dim}\left(A^{y}{ }_{x}\right)=\mathrm{A} \Theta^{-1} \mathrm{~s} & \operatorname{dim}\left(A^{y}{ }_{y}\right)=\mathrm{A} .
\end{array}
$$

The intrinsic dimension of the tensor $\boldsymbol{A}$ is A. The expansion (7) shows that this dimension is independent of the coordinate system, by construction - such expansion could be done in any other coordinate system, and the left side would be the same. The effect of coordinate transformations is examined more in detail in $\S 5$. The intrinsic dimension A is determined by the physical and operational meaning of the tensor $\boldsymbol{A}$; see $\S \S 9,10$ for concrete examples. Together with the dimensions of the coordinates it determines the dimensions of the components, eqs (8), which need not be all equal.

This simple example should have disclosed the main points of dimensional analysis on manifolds, which will now be discussed in more generality. In the derivation above we silently adopted a couple of natural conventions: for example, that the tensor product behaves similarly to multiplication with regard to dimensions. Such conventions are briefly discussed in $\S 12$.

\section{Coordinates}

From a physical point of view a coordinate is just a function that associates values of some physical quantity with the events in a region (the domain of the coordinate chart) of spacetime. Together with the other coordinates such function allows us to uniquely identify every event within that region. Any physical quantity will do: the distance from something, the time elapsed since something, an angle, an energy density, the strength of a magnetic flux, a temperature, and so on. A coordinate can thus have any dimension: length $L$, time $T$, angle 1 , temperature $\Theta$, magnetic flux $\Phi:=\mathrm{ML}^{2} \mathrm{~T}^{-2} \mathrm{I}^{-1}$, and so on.

The functional relation between two sets of coordinates must of course be dimensionally consistent. For example, if $\operatorname{dim}\left(x^{0}\right)=\mathrm{T}$ and 
$\operatorname{dim}\left(x^{1}\right)=\mathrm{L}$, and we introduce a new coordinate $y\left(x^{0}, x^{1}\right)$ with dimension $\Phi$, additive in the previous two, then we must have $y=a x^{0}+b x^{1}$ with $\operatorname{dim}(a)=\Phi \mathrm{T}^{-1}$ and $\operatorname{dim}(b)=\Phi \mathrm{L}^{-1}$.

\section{Tensors: intrinsic dimension and components' dimensions}

Consider a system of coordinates $\left(x^{i}\right)$ with dimensions $\left(\mathrm{X}_{i}\right)$, and the ensuing sets of covector fields $\left(\mathrm{d} x^{i}\right)$ and of vector fields $\left(\frac{\partial}{\partial x^{i}}\right)$, bases for the cotangent and tangent spaces. Their tensor products are bases for the tangent spaces of higher tensor types.

The differential $\mathrm{d} x^{i}$ traditionally has the same dimension as $x^{i}$ : $\operatorname{dim}\left(\mathrm{d} x^{i}\right)=\mathrm{X}_{i}$, and the vector $\frac{\partial}{\partial x^{i}}$ traditionally has the inverse dimension: $\operatorname{dim}\left(\frac{\partial}{\partial x^{i}}\right)=X_{i}^{-1}$.

For our discussion let's take a concrete example: a contra-co-variant tensor field $\boldsymbol{A} \equiv \boldsymbol{A}^{*}$. . The discussion generalizes to tensors of other types in an obvious way.

The tensor $\boldsymbol{A}$ can be expanded in terms of the basis vectors and covectors, as in $\S 2$ and in the example of $\S 3$ :

$$
\boldsymbol{A}=A^{i}{ }_{j} \frac{\partial}{\partial x^{i}} \otimes \mathrm{d} x^{j} \equiv A^{0}{ }_{0} \frac{\partial}{\partial x^{0}} \otimes \mathrm{d} x^{0}+A^{0}{ }_{1} \frac{\partial}{\partial x^{0}} \otimes \mathrm{d} x^{1}+\cdots .
$$

Each function

$$
A_{j}^{i}:=\boldsymbol{A}\left(\mathrm{d} x^{i}, \frac{\partial}{\partial x^{j}}\right) \equiv \operatorname{tr}_{12} \operatorname{tr}_{34}\left(\boldsymbol{A}^{\bullet} \cdot \otimes \mathrm{d} x^{i} \otimes \frac{\partial}{\partial x^{j}}\right) \equiv \mathrm{d} x^{i} \boldsymbol{A} \frac{\partial}{\partial x^{j}}
$$

is a component of the tensor in this coordinate system.

To make dimensional sense, all terms in the sum (9) must have the same dimension. This is possible only if the generic component $A^{i}{ }_{j}$ has dimension

$$
\operatorname{dim}\left(A^{i}{ }_{j}\right)=\mathrm{A} \mathrm{X}_{i} \mathrm{X}_{j}^{-1}
$$

where $\mathrm{A}$ is common to all components. In fact, the $\mathrm{X}_{i} \mathrm{X}_{j}^{-1}$ term cancels the $\mathrm{X}_{i}^{-1} \mathrm{X}_{j}$ term coming from $\frac{\partial}{\partial x^{i}} \otimes \mathrm{d} x^{j}$ in the sum (9), and each summand therefore has dimension $\mathrm{A}$. 
The generalization of the formula above to tensors of other types is obvious:

$$
\operatorname{dim}\left(A_{k l \ldots}^{i j \ldots}\right)=\mathrm{A} \mathrm{X}_{i} \mathrm{X}_{j} \ldots \mathrm{X}_{k}^{-1} \mathrm{X}_{l}^{-1} \ldots,
$$

where the ordering of the indices doesn't matter. Clearly the components can have different dimensions ${ }^{13}$. What matters is that the sum (9) be dimensionally consistent.

The dimension A, which is also the dimension of the sum (9), I'll call the intrinsic dimension of the tensor $\boldsymbol{A}$, and we write

$$
\operatorname{dim}(\boldsymbol{A})=\mathrm{A} .
$$

This dimension is independent of any coordinate system. It reflects the physical or operational ${ }^{14}$ meaning of the tensor. We shall see an example of such an operational analysis in $\S \S 9,10$ for the metric and stress-energy-momentum tensors.

The notion of intrinsic dimension was introduced by Dorgelo and Schouten ${ }^{15}$ under the name 'absolute dimension'. I find the adjective 'intrinsic' more congruous to modern terminology and less prone to suggest spurious connections with absolute values. In the following I'll drop the adjective 'intrinsic' when it is clear from the context.

Different coordinate systems lead to different dimensions of the components of a tensor $\boldsymbol{A}$, but the intrinsic dimension of the tensor remains the same. Formula (12) for the dimensions of the components is consistent under changes of coordinates. For example, in new coordinates $\left(\bar{x}^{k}\right)$ with dimensions $\left(\bar{X}_{k}\right)$, the new components of $\boldsymbol{A}$ are

$$
\bar{A}_{l}^{k}=A_{j}^{i} \frac{\partial \bar{x}^{k}}{\partial x^{i}} \frac{\partial x^{j}}{\partial \bar{x}^{l}}
$$

and a quick check shows that $\operatorname{dim}\left(\bar{A}^{k}{ }_{l}\right)=\mathrm{A} \overline{\mathrm{X}}_{k} \overline{\mathrm{X}}_{l}^{-1}$, consistently with the general formula (12).

If in eq. (14), relating intrinsic and component dimensions, all coordinates have equal dimensions, $\mathrm{X}_{i}=\mathrm{X}$ for all $i$, then all components also have equal dimensions. So if we use a system of coordinates having equal

13 cf. the discussion in Synge 1960a § IV.5 p. $179 . \quad 14$ Bridgman 1958; see also Synge 1960b § A.2; Truesdell \& Toupin 1960 § A.3-4. 15 Dorgelo \& Schouten 1946; Schouten 1989 ch. VI. 
dimensions, the components of any tensor must also have equal dimensions. This justifies common practice in the literature.

Choosing coordinates of different dimensions, however, has several advantages. First, it allows us to use dimensional analysis as a heuristic tool to determine the variance type of a tensor; we'll see an example in $\S 10$. Second, it can lead to components with familiar dimensions. For example, if we use a timelike coordinate of dimension $\mathrm{T}$ and spacelike coordinates of dimension $\mathrm{L}$, then the components of the (co-contravariant) stress-energy-momentum tensor have the familiar dimensions of energy density, surface energy-flux density, momentum density, and pressure, with no $c$ factors involved; see again $\S 10$.

\section{Tensor operations}

By the reasoning of the previous section, which simply applies standard dimensional considerations to the basis expansion (9), it's easy to find the resulting intrinsic dimension of various operations and operators on tensors and tensor fields.

Here is a summary of the dimensional rules for the main differentialgeometric operations and operators, except for the covariant derivative, the metric, and related tensors, discussed more in depth in $\$ \S 8-9$ below. Some of these rules are actually definitions or conventions, as briefly discussed in their description. The others can be proved; I only give proofs for some of them, leaving the other proofs as an exercise. For reference, in brackets I give the section of Choquet-Bruhat et al. (1996) where these operations are defined.

- The tensor product [III.B.5] multiplies dimensions:

$$
\operatorname{dim}(\boldsymbol{A} \otimes \boldsymbol{B})=\operatorname{dim}(\boldsymbol{A}) \operatorname{dim}(\boldsymbol{B}) .
$$

This is actually a definition or convention. We tacitly used this rule already in the example of $\S 3$ and in $\S 5$ for the coordinate expansion (9). It is a natural definition, because for tensors of order 0 (functions) the tensor product is just the ordinary product, and the dimension of a product is the product of the dimensions. This definition doesn't lead to inconsistencies. 
- The contraction [III.B.5] or trace of the $\alpha$ th and $\beta$ th slots of a tensor has the same dimension as the tensor:

$$
\operatorname{dim}\left(\operatorname{tr}_{\alpha \beta} \boldsymbol{A}\right)=\operatorname{dim}(\boldsymbol{A}) .
$$

Note that the formula above only holds without raising or lowering indices; see $\S 9.2$ for those operations.

This operation can be traced back to the duality of vectors and covectors mentioned in $\S 2$ : a covector field $\boldsymbol{\omega}$ operates on a vector field $v$ to yield a function $f=\boldsymbol{\omega}(\boldsymbol{v})$. Also in this case we have that $\operatorname{dim}(f)=$ $\operatorname{dim}(\boldsymbol{\omega}) \operatorname{dim}(v)$ by definition or convention, and the rule (16) follows from this convention. Also in this case this convention seems very natural, owing to the linearity properties of the trace, and doesn't lead to inconsistencies.

- The transposition ${ }^{16}$ of the $\alpha$ th and $\beta$ th slots of a tensor has the same dimension as the tensor:

$$
\operatorname{dim}\left(\boldsymbol{A}^{\top \alpha \beta}\right)=\operatorname{dim}(\boldsymbol{A}) .
$$

- The Lie bracket [III.B.3] of two vectors has the product of their dimensions:

$$
\operatorname{dim}([\boldsymbol{u}, \boldsymbol{v}])=\operatorname{dim}(\boldsymbol{u}) \operatorname{dim}(\boldsymbol{v}) .
$$

In fact, in coordinates $\left(x^{i}\right)$ the bracket can be expressed as

$$
[u, v]=\left(u^{j} \frac{\partial v^{i}}{\partial x^{j}}-v^{j} \frac{\partial u^{i}}{\partial x^{j}}\right) \frac{\partial}{\partial x^{i}},
$$

and equating the dimensions of the left and right sides, considering that

$$
\operatorname{dim}\left(u^{i}\right)=\operatorname{dim}(u) X_{i}, \quad \operatorname{dim}\left(v^{i}\right)=\operatorname{dim}(v) X_{i},
$$

we find again that all $\mathrm{X}$ terms cancel out, leaving the result (18).

- The pull-back [III.A.2], tangent map [III.B.1], and push-forward of a map $F$ between manifolds don't change the dimensions of the tensors they map. The reason, evident from their definitions, is that they all rest on the pull-back of a function: $F^{*}(f):=f \circ F$, which, being a composition, has the same dimension as the function.

16 called “building an isomer" by Schouten 1954 § I.3 p. 13; 1989 § II.4 p. 20. 
- The Lie derivative [III.C.2] of a tensor with respect to a vector field has the product of the dimensions of the two:

$$
\operatorname{dim}\left(\mathrm{L}_{v} \boldsymbol{A}\right)=\operatorname{dim}(\boldsymbol{v}) \operatorname{dim}(\boldsymbol{A}) .
$$

Regarding operations and operators on differential forms:

- The exterior product [IV.A.1] of two differential forms multiplies their dimensions:

$$
\operatorname{dim}(\boldsymbol{\omega} \wedge \boldsymbol{\xi})=\operatorname{dim}(\boldsymbol{\omega}) \operatorname{dim}(\boldsymbol{\xi}) .
$$

- The interior product [IV.A.4] (also called 'inner', 'dual', or 'dot' product) of a vector and a covector multiplies their dimensions:

$$
\operatorname{dim}(v \cdot \boldsymbol{w})=\operatorname{dim}(\boldsymbol{v}) \operatorname{dim}(\boldsymbol{w}) .
$$

This equation also holds for the generalized interior product ${ }^{17}$ of a multi-vector $v$ and a multi-covector $\boldsymbol{\omega}$. The interior product is also often

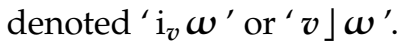

- The exterior derivative [IV.A.2] of a form has the same dimension of the form:

$$
\operatorname{dim}(\mathrm{d} \boldsymbol{\omega})=\operatorname{dim}(\boldsymbol{\omega}) .
$$

This can be proven using the identity $\mathrm{d}(\boldsymbol{v} \cdot \boldsymbol{\omega})+v \cdot(\mathrm{d} \boldsymbol{\omega})=\mathrm{L}_{v} \boldsymbol{\omega}$ or similar identities ${ }^{18}$ together with eqs (21) and (23).

- The integral [IV.B.1] of a form over a submanifold (or more generally a chain) $M$ has the same dimension as the form:

$$
\operatorname{dim}\left(\int_{M} \omega\right)=\operatorname{dim}(\omega)
$$

The reason is that the integral of a form over a submanifold or chain ultimately rests on the standard definition of integration on the real line $^{19}$, which satisfies the dimensional rule above. In fact, the integral is invariant with respect to reparameterizations of the chain; it depends

17 Deschamps 1970; 1981 Appendices; Lindell 2004; Truesdell \& Toupin 1960 § F.I.267; Misner et al. 1973 Box 4.1, item 4; see also Porta Mana 2019a. 18 Curtis \& Miller 1985 ch. 9 p. 180 Theorem 9.78; Abraham et al. $1988 \S 6.4$ Theorem 6.4.8. $\quad 19$ e.g. Choquet-Bruhat et al. $1996 \S \S$ IV.B.1-2; de Rham 1984 § 5 p. 21, §6 p. 24; Abraham et al. 1988 § 7.1; Boothby $2003 \S$ VI.2. 
only on its image (some texts ${ }^{20}$ even define chains as equivalence classes determined by their image).

All rules above extend in obvious ways to tensor densities, and apply regardless whether the objects have straight or twisted orientations.

\section{Curves and integral curves}

Consider a curve into spacetime $C: s \mapsto P(s)$, with the parameter $s$ having some dimension $\operatorname{dim}(s)=\mathrm{S}$.

If we consider the events of the spacetime manifold as dimensionless quantities, then the dimension of the tangent or velocity vector $\dot{C}$ to the curve is

$$
\operatorname{dim}(\dot{C})=\mathrm{S}^{-1}
$$

owing to the definition ${ }^{21}$

$$
\dot{C}:=\frac{\partial\left(x^{i} \circ C\right)}{\partial s} \frac{\partial}{\partial x^{i}} .
$$

Note an important consequence of this fact. Given a vector field $v$ we say that $C$ is an integral curve for it if

$$
v=\dot{C}
$$

at all events $C(s)$ in the image of the curve (or $v_{C(s)}=\dot{C}_{C(s)}$ in standard differential-geometric notation ${ }^{22}$ ). From the point of view of dimensional analysis this definition is only valid if $v$ has dimension $\mathrm{S}^{-1}$. If $v$ and $s^{-1}$ have different dimensions - a case which could happen for physical reasons - the condition (28) must be modified into $v=k \dot{C}$, where $k$ is a dimensionful constant. This is equivalent to considering an affine and dimensional reparameterization of $C$.

Worldlines and their 4-velocities are discussed in $\S 9.3$.

\section{Connection, covariant derivative, curvature tensors}

Consider an arbitrary connection ${ }^{23}$ with covariant derivative $\nabla$. For the moment we don't assume the presence of any metric structure.

20 e.g. Martin 2004 § 10.4; Fecko $2006 \S 7.3 . \quad 21$ Choquet-Bruhat et al. 1996 § III.B.1; Boothby $2003 \S$ IV.(1.9). $\quad 22$ Choquet-Bruhat et al. $1996 \S$ III.B.1. $\quad 23$ Choquet-Bruhat et al. $1996 \S$ V.B. 
The covariant derivative of the product $f v$ of a function and a vector satisfies $^{24}$

$$
\nabla(f v)=\mathrm{d} f \otimes v+f \nabla v .
$$

The first summand, from formulae (24) and (15), has dimension $\operatorname{dim}(f) \operatorname{dim}(v)$; for dimensional consistency this must also be the dimension of the second summand. Thus

$$
\operatorname{dim}(\nabla v)=\operatorname{dim}(v) .
$$

It follows that the directional covariant derivative $\nabla_{u}$ has dimension

$$
\operatorname{dim}\left(\nabla_{\boldsymbol{u}} \boldsymbol{v}\right)=\operatorname{dim}(\boldsymbol{u}) \operatorname{dim}(\boldsymbol{v}),
$$

and by its derivation properties ${ }^{25}$ we see that formula (30) extends from vectors to tensors of arbitrary type.

In the coordinate system $\left(x^{i}\right)$ the action of the covariant derivative is carried by the connection coefficients or Christoffel symbols $\left(\Gamma_{j k}^{i}\right)$ defined by

$$
\nabla \frac{\partial}{\partial x^{k}}=\Gamma_{j k}^{i} \mathrm{~d} x^{j} \otimes \frac{\partial}{\partial x^{i}} .
$$

From this equation and eqs (15), (30) it follows that an individual coefficient has dimension

$$
\operatorname{dim}\left(\Gamma_{j k}^{i}\right)=\mathrm{X}_{i} \mathrm{X}_{j}^{-1} \mathrm{X}_{k}^{-1}
$$

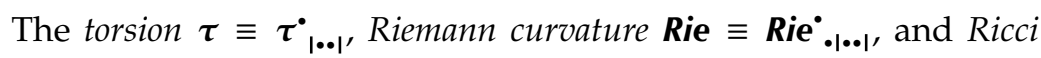
curvature $\boldsymbol{R i c} \equiv \boldsymbol{R i c}$. . tensors are defined by ${ }^{26}$

$$
\begin{gathered}
\tau(u, v):=\nabla_{u} v-\nabla_{v} u-[u, v], \\
\operatorname{Rie}(w ; u, v):=\nabla_{u} \nabla_{v} w-\nabla_{v} \nabla_{u} w-\nabla_{[u, v]} w, \\
\text { Ric.. }_{.=}=\operatorname{tr}_{13} \operatorname{Rie}_{\text {.|॰. }}^{\cdot} .
\end{gathered}
$$

From these definitions and the results of $\S 6$ we find the dimensional requirements

$$
\begin{aligned}
& \operatorname{dim}\left(\boldsymbol{\tau}_{|\bullet \bullet|}{ }^{\circ}\right) \operatorname{dim}(\boldsymbol{u}) \operatorname{dim}(\boldsymbol{v})=\operatorname{dim}(\boldsymbol{u}) \operatorname{dim}(\boldsymbol{v}), \\
& \operatorname{dim}\left(\boldsymbol{R i e}_{\bullet \mid \cdot \boldsymbol{~} \boldsymbol{|}}\right) \operatorname{dim}(\boldsymbol{w}) \operatorname{dim}(\boldsymbol{u}) \operatorname{dim}(\boldsymbol{v})=\operatorname{dim}(\boldsymbol{w}) \operatorname{dim}(\boldsymbol{u}) \operatorname{dim}(\boldsymbol{v}), \\
& \operatorname{dim}\left(\text { Ric.. }_{\text {. }}\right)=\operatorname{dim}\left(\boldsymbol{R i e}_{\bullet|\cdot \bullet|}\right),
\end{aligned}
$$

\footnotetext{
24 Choquet-Bruhat et al. $1996 \S$ V.B.1.

26 Choquet-Bruhat et al. $1996 \S$ V.B.1.

25 Choquet-Bruhat et al. 1996 § V.B.1 p. 303.
} 
which imply that the torsion, Riemann curvature, and Ricci curvature tensors are dimensionless:

$$
\operatorname{dim}\left(\boldsymbol{\tau}_{|\bullet \bullet|}^{\cdot}\right)=\operatorname{dim}\left(\boldsymbol{R i e}_{\bullet|\bullet| l \mid}\right)=\operatorname{dim}\left(\boldsymbol{R i c}_{. .}\right)=1 .
$$

This result is sensible because the notion of local parallelism, which these tensors express, doesn't involve any notion of distance or angle ${ }^{27}$. The exact contra- and co-variant primitive type of these tensors is very important in the equations above. If a metric tensor is also introduced and used to raise or lower any indices of these tensors, the resulting tensors will have different dimensions; see $§ 9.2$.

Misner et al. ${ }^{28}$ say that "curvature", by which they seem to mean the Riemann tensor, has dimension $\mathrm{L}^{-2}$. This statement is seemingly at variance with the dimensionless results (40). But I believe that Misner et al. refer to the components of the Riemann tensor in specific coordinates of dimension L. In such specific coordinates every component $\mathrm{Rie}^{i}{ }_{j k l}$ has dimension $L^{-2}$, according to the general formula (12), if and only if the intrinsic dimension of $\boldsymbol{R i e}$ is unity, $\operatorname{dim}(\boldsymbol{R i e})=1$. So I believe that Misner et al.'s statement actually agrees with the results (40). This possible misunderstanding shows the importance of distinguishing between the intrinsic dimension, which doesn't depend on any specific coordinate choice, and component dimensions, which do.

The formulae above are also valid if a metric is defined and the connection is compatible with it, see $\S 9.4$ below.

\section{Metric and related tensors and operations}

\subsection{Intrinsic dimensions: two choices}

Let us now consider a metric tensor $\mathbf{g} \equiv \mathbf{g} .$. . What is its intrinsic dimension $\operatorname{dim}(\mathbf{g})$ ? The literature offers two choices; both can be motivated by the operational meaning of the metric.

Consider a (timelike) worldline $s \mapsto C(s), s \in[a, b]$, between events $C(a)$ and $C(b)$. The metric tells us the proper time $\Delta t$ elapsed for an observer having that worldline, according to the formula

$$
\Delta t=\int_{a}^{b} \sqrt{|\boldsymbol{g}[\dot{C}(s), \dot{C}(s)]|} \mathrm{d} s .
$$

27 cf. Porta Mana 2019b. $\quad{ }^{28}$ Misner et al. 1973 p. 35. 
From the results of $\S 6$ this formula implies that $\mathrm{T} \equiv \operatorname{dim}(\Delta t)=\sqrt{\operatorname{dim}(\text { g.. })}$, independently of the dimension of the parameter $s$, and therefore

$$
\operatorname{dim}(\text { g.. })=\mathrm{T}^{2} .
$$

Most authors ${ }^{29}$, however, prefer to include a dimensional factor $1 / c$ in the definition (41):

$$
\Delta t=\int_{a}^{b} \frac{1}{c} \sqrt{|g[\dot{C}(s), \dot{C}(s)]|} \mathrm{d} s,
$$

thus obtaining

$$
\operatorname{dim}(\text { g.. })=\mathrm{L}^{2} .
$$

The choice (44) is also supported by the traditional expression for the "line element $d s^{2}$ " as it appears in many works:

$$
d s^{2}=-c^{2} d t^{2}+d x^{2}+d y^{2}+d z^{2},
$$

sometimes with opposite sign. If the coordinates $(t, x, y, z)$ have the dimensions suggested by their symbols, this formula has dimension $\mathrm{L}^{2}$, so that if we interpret " $d s^{2 \prime \prime}$ as $\mathbf{g}$.. we find $\operatorname{dim}($ g.. $)=\mathrm{L}^{2}$. The lineelement expression above often has an ambiguous differential-geometric meaning, however, because it may also represent the metric applied to some unspecified vector, that is, $d s^{2}=\boldsymbol{g}(\boldsymbol{v}, \boldsymbol{v})$ with $\boldsymbol{v}$ left unspecified ${ }^{30}$. In this case we have

$$
\mathrm{L}^{2}=\operatorname{dim}(\mathbf{g}) \operatorname{dim}(v)^{2}
$$

and the dimension of $\boldsymbol{g}$ is ambiguous or undefined, because the vector $\boldsymbol{v}$ could have any dimension.

The standard choices for $\operatorname{dim}(\boldsymbol{g})$ are thus $\mathrm{L}^{2}$ or $\mathrm{T}^{2}$; the corresponding metric tensors differ by a factor $c^{2}$.

The choice $\operatorname{dim}(\mathbf{g}):=\mathrm{T}^{2}$, used for example by McVittie, Synge, Kilmister $^{31}$, has some advantages for the definition of the co-variant 4 -velocity, discussed in $\S 9.3$. It could be motivated on operational grounds for reasons discussed by Synge and Bressan ${ }^{32}$. Synge gives a vivid summary: ${ }^{33}$

29 e.g. Fock 1964 § V.62 eq. (62.02); Curtis \& Miller 1985 ch. 11 eq. (11.21); Rindler 1986 $\S 5.3$ eq. (5.6); Hartle 2003 ch. 6 eq. (6.24). $\quad 30$ cf. Misner et al. 1973 Box 3.2 D p. 77.

31 McVittie 1965 § 4.1; Synge 1960a § IV.5; Kilmister 1973 ch. II p. $25 . \quad 32$ Synge 1960a §§ III.2-4; Bressan 1978 § 15, $18 . \quad 33$ Synge 1960a § III.3 pp. 108-109. 
We are now launched on the task of giving physical meaning to the Riemannian geometry [...]. It is indeed a Riemannian chronometry rather than geometry, and the word geometry, with its dangerous suggestion that we should go about measuring lengths with yardsticks, might well be abandoned altogether in the present connection

In fact, to measure the proper time $\Delta t$ defined above we only need to ensure that a clock has the worldline $C$, and then take the difference between the clock's final and initial times. If $C$ is spacelike instead, the measurement of its proper length, still defined by the integral (41) apart from a dimensional constant, is more involved. It requires dividing the curve into very short pieces, and having specially-chosen observers (with 4-velocities orthogonal to the pieces) measure each piece. To measure each short piece, each observer uses radar distance, sending a light signal which bounces back at the end of the piece, and timing how long it takes to come back ${ }^{34}$. Even if rigid rods are used, their calibration still relies on a measurement of time - this is also reflected in the current definition of the standard metre ${ }^{35}$. Thus the measurement of length seems to ultimately rely on the measurement of time. It could be objected, however, that the laws of light propagation depend on the metric tensor, which connects the Faraday and Maxwell tensors ${ }^{36}$, so this reasoning could be circular.

The other choice, $\operatorname{dim}(\boldsymbol{g}):=\mathrm{L}^{2}$, is by far the most common. It has the merit that the projection of the metric onto a spacelike hypersurface also has dimension $L^{2}$, which is sensible from a Newtonian point of view. Such projections are at the heart of $3+1$ formulations of general relativity ${ }^{37}$ and also of covariant formulations of Newtonian mechanics ${ }^{38}$.

In the following we shall see how both choices affect several dimensional results.

\subsection{Inverse metric, index raising and lowering, proper volume element}

The metric $\mathbf{g}$ can be considered as an operator mapping vectors $\boldsymbol{v}$ to covectors $\boldsymbol{\omega}$, which we can compactly write as $\boldsymbol{\omega}=\mathbf{g} \boldsymbol{v}$ as discussed in

\footnotetext{
34 Frankel 1979 ch. 2; Landau \& Lifshitz 1996 § $84 . \quad 35$ вIPM 1983 p. 98 ; Giacomo 1984 p. 25. 36 Truesdell \& Toupin 1960 ch. F.III; Misner et al. 1973 ch. II.4; Puntigam et al. 1997; Hehl \& Obukhov 2001; 2005b. 37 Gourgoulhon 2012; Alcubierre 2008; Misner et al. 1973 ch. 21; Wilson \& Mathews 2007; Smarr \& York 1978; York 1979; Smarr et al. 1980; I thank I. Bengtsson for this remark.

38 Truesdell \& Toupin 1960 §§ B.II.152-154, D.II.203-205, D.V.238, F.IV.285-289; Marsden \& Hughes 1994 § 2.4.
} 
$\S 2$. The inverse metric tensor $\mathbf{g}^{-1} \equiv \mathbf{g}^{-1} \cdot \boldsymbol{\bullet}$ is then defined by

$$
\mathrm{gg}^{-1}=\mathrm{id}_{\bullet}^{\bullet}, \quad \mathrm{g}^{-1} \mathrm{~g}=\mathrm{id}^{\bullet},
$$

where id. $: \boldsymbol{\omega} \mapsto \boldsymbol{\omega}$ is the dimensionless identity operator (also a tensor) on the cotangent space, and id $^{\bullet}$. on the tangent space. Hence

$$
\operatorname{dim}\left(\mathbf{g}^{-1}\right)=\operatorname{dim}(\mathbf{g})^{-1} .
$$

The operation of raising or lowering an index of a tensor represents a contraction of the tensor product of that tensor with the metric or the metric inverse, for example $\underline{\boldsymbol{A}} .$. $\boldsymbol{A}^{\boldsymbol{*}}$., and similarly for tensors of other types. Therefore every lowering of a tensor's index multiplies its dimension by $\operatorname{dim}(\mathbf{g})$, and every rising divides it by $\operatorname{dim}(\mathbf{g})$ :

$$
\begin{aligned}
& \operatorname{dim}\left(\underline{\boldsymbol{A}}_{\ldots} \ldots\right)=\operatorname{dim}\left(\boldsymbol{A}_{\ldots} \ldots \ldots\right) \operatorname{dim}(\mathbf{g}) \\
& \operatorname{dim}\left(\overline{\boldsymbol{B}}_{\ldots} \cdot \ldots\right)=\operatorname{dim}\left(\boldsymbol{B}_{\ldots \cdot \ldots}\right) \operatorname{dim}(\boldsymbol{g})^{-1} .
\end{aligned}
$$

The volume element in a four-dimensional spacetime is a twisted 4 -form uniquely determined by the metric tensor ${ }^{39}$. Its only non-zero component is equal to the square root of the determinant of the components $\left(g_{i j}\right)$ of the metric:

$$
\sqrt{\left|\operatorname{det}\left(g_{i j}\right)\right|} \mathrm{d} x^{0} \wedge \mathrm{d} x^{1} \wedge \mathrm{d} x^{2} \wedge \mathrm{d} x^{3} .
$$

Here $\mathrm{d} x^{0} \wedge \mathrm{d} x^{1} \wedge \mathrm{d} x^{2} \wedge \mathrm{d} x^{3}$ actually has a twisted orientation ${ }^{40}$ (the coordinate transformation of the non-zero component includes the sign of the Jacobian), which in this case means that it has no screw-sense orientation at all, only an abstract ' + ' orientation. For this reason a globally non-vanishing volume element can be defined on orientable and non-orientable manifolds alike. From the results of $\S 6$ it can be shown that the 4 -form above has intrinsic dimension $\operatorname{dim}(g)^{2}$ (in an $n$-dimensional spacetime it has dimension $\left.\operatorname{dim}(g)^{n / 2}\right)$. It's convenient

39 De Rham 1984 § V.24; Choquet-Bruhat et al. 1996 § V.A.4; Abraham et al. 1988 § 6.2.

40 Frankel 1979 ch. 6 p. 60, ch. 9. 
to multiply it by a power of $c$ and to define the proper volume element $\gamma \equiv \gamma_{1 \ldots . .1}$ as follows:

$$
\gamma:=\left\{\begin{array}{ll}
\frac{1}{c} \sqrt{\left|\operatorname{det}\left(g_{i j}\right)\right|} \mathrm{d} x^{0} \wedge \mathrm{d} x^{1} \wedge \mathrm{d} x^{2} \wedge \mathrm{d} x^{3} & \text { if } \operatorname{dim}(\mathbf{g}):=\mathrm{L}^{2} \\
c^{3} \sqrt{\left|\operatorname{det}\left(g_{i j}\right)\right|} \mathrm{d} x^{0} \wedge \mathrm{d} x^{1} \wedge \mathrm{d} x^{2} \wedge \mathrm{d} x^{3} & \text { if } \operatorname{dim}(\mathbf{g}):=\mathrm{T}^{2}
\end{array} .\right.
$$

As a consequence we have

$$
\operatorname{dim}\left(\gamma_{1 \ldots . .1}\right)=\mathrm{L}^{3} \mathrm{~T}
$$

independently of whether $\operatorname{dim}(\boldsymbol{g})$ equals $L^{2}$ or $T^{2}$. This convention has several advantages, and implies that the hypervolume of a fourdimensional region, given by the integral of $\gamma$, also has dimension $\mathrm{L}^{3} \mathrm{~T}$, see eq. (25) - which is a reasonable result for a space $\left(\mathrm{L}^{3}\right)$ time $(\mathrm{T})$ region.

In general the metric $\boldsymbol{g}$ induces volume, area, and line elements on three-, two-, and one-dimensional regions. It is convenient to multiply these elements by appropriate powers of $c$ so that the region's volume has intuitive dimensions, such as $\mathrm{L}^{3}$ for a spacelike three-dimensional region and LT for a timelike two-dimensional one. Indeed the definition of proper time (43) does exactly this, including a factor $1 / c$ in the induced line element on a timelike curve.

The inverse proper volume element is the 4-vector field $\gamma^{-1}$, with twisted orientation, having unit generalized inner product with the proper volume element: $\gamma^{-1} \cdot \gamma=1$. Its intrinsic dimension is therefore

$$
\operatorname{dim}\left(\gamma^{-1|\cdots \cdot \bullet|}\right)=\operatorname{dim}(\gamma)^{-1} \equiv \mathrm{L}^{-3} \mathrm{~T}^{-1}
$$

again independently of whether $\operatorname{dim}(g)$ equals $L^{2}$ or $T^{2}$. Note that the inverse proper volume element is dimensionally and numerically different from the tensor obtained by raising all indices of $\gamma$.

The proper volume element appears in the various definitions of the star operator ${ }^{41}$ on covectors and forms. This operator usually acts by first rising all indices of a covector and then taking the generalized inner product (see $\S 6$ ) with the proper volume element. For example, for a 2-covector $\omega \equiv \omega_{|\cdot \bullet|}$

$$
* \omega:=\left(\mathbf{g}^{-1} \omega \mathbf{g}^{-1}\right) \cdot \gamma,
$$

with $\mathbf{g}^{-1}$ appearing twice in this specific case. From the general definition it's clear that the star operator's effect on the dimension depends on the

41 e.g. Choquet-Bruhat et al. 1996 § V.A.4; Misner et al. 1973 Box 4.3; Burke 1987 § IV.24. 
degree of the form it operates on. I personally prefer to avoid the star operator and to explicitly use the inner product with the proper volume element ${ }^{42}$.

\subsection{Four-velocity and projector onto it}

The worldline of an observer or of a small body is a timelike curve $C: \tau \mapsto P(\tau)$ into spacetime, parameterized by the proper time $\tau$. If we assume $\operatorname{dim}(\tau)=\mathrm{T}$, then according to the discussions in $\S \S 7,9.1,9.2$ the condition that the curve's parameter should beat the proper time leads to two different normalization conditions for the 4-velocity tangent vector $U \equiv U^{\bullet}:=\dot{C}$, depending on the choice of dimension for the metric:

$$
\begin{aligned}
-\frac{1}{c^{2}} \boldsymbol{U}(\tau) \boldsymbol{g} \boldsymbol{U}(\tau)=1 & \text { if } \operatorname{dim}(\boldsymbol{g}):=\mathrm{L}^{2}, \\
-\boldsymbol{U}(\tau) \boldsymbol{g} \boldsymbol{U}(\tau)=1 & \text { if } \operatorname{dim}(\boldsymbol{g}):=\mathrm{T}^{2},
\end{aligned}
$$

either equation is dimensionless under its specific condition. Independently of normalization conditions the 4 -velocity has intrinsic dimension $\operatorname{dim}\left(\boldsymbol{U}^{\bullet}\right)=\mathrm{T}^{-1}$.

Both conditions lead to the same expression for the 4-velocity in a system of rectangular Cartesian coordinates $(t, x, y, z)$ with $\operatorname{dim}(t, x, y, z)=$ $(T, L, L, L)$, adapted to an inertial observer:

$$
U=\beta \partial_{t}+\beta V^{r} \partial_{r}, \quad \beta:=1 / \sqrt{1-V^{2} / c^{2}}
$$

where $\beta$ is the (dimensionless) Lorentz contraction factor and $V^{r}, r \in$ $\{x, y, z\}$, are the components of the coordinate 3-velocity $\boldsymbol{V}$.

Care must be taken with the covariant 4-velocity, usually obtained by lowering the index of $\boldsymbol{U}$. It is convenient to define it as

$$
\underline{\boldsymbol{U}}:=\left\{\begin{array}{ll}
\frac{1}{c^{2}} \boldsymbol{U g} & \text { if } \operatorname{dim}(\boldsymbol{g}):=\mathrm{L}^{2} \\
\boldsymbol{U} \boldsymbol{g} & \text { if } \operatorname{dim}(\boldsymbol{g}):=\mathrm{T}^{2}
\end{array} .\right.
$$

Either definition leads to the same expression in rectangular Cartesian inertial coordinates, and also to the property $\boldsymbol{U} \underline{U}=-1$, with $\operatorname{dim}(\underline{\boldsymbol{U}})=$ $\operatorname{dim}(\boldsymbol{U})^{-1}=\mathrm{T}^{-1}$, independently of the choice of dimension for the metric. This property is especially useful in $3+1$ formulations of general relativity,

42 cf. Bossavit 1991 §§ 4.1-2. 
where $\boldsymbol{U} \otimes \underline{U}$ acts as a projector onto the proper-time axis. If you use $\operatorname{dim}(\boldsymbol{g}):=\mathrm{L}^{2}$ and define the covariant 4-velocity as $\boldsymbol{U} \boldsymbol{g}$ instead, then keep in mind that such a projector requires an extra factor $1 / c^{2}$.

Alternatively we can stipulate that when $\operatorname{dim}(g):=\mathrm{L}^{2}$ the proper time is actually a length, $\operatorname{dim}(\tau):=\mathrm{L}$. Then the $c^{2}$ factors in the formulae above disappear, the special coordinate expression (54) must be replaced by $\boldsymbol{U}=c \beta \partial_{t}+c \beta V^{r} \partial_{r}$, and we have $\boldsymbol{U} \underline{\boldsymbol{U}}=-1$ with $\operatorname{dim}(\underline{\boldsymbol{U}})=\operatorname{dim}(\boldsymbol{U})^{-1}=$ $\mathrm{L}^{-1}$ in this case.

\subsection{Induced connection and Einstein tensor}

The formulae for the covariant derivative (30), connection coefficients (33), and curvature tensors (40) remain valid for a connection compatible with the metric. In this case the connection coefficients can be obtained from the metric by the formulae ${ }^{43}$

$$
\Gamma_{j k}^{i}=\frac{1}{2}\left(\frac{\partial}{\partial x^{k}} g_{j l}+\frac{\partial}{\partial x^{j}} g_{k l}-\frac{\partial}{\partial x^{l}} g_{j k}\right) g^{l i},
$$

and it's easily verified that the dimensions of these coefficients given in eq. (33) still hold, as do the results for the curvature tensors (40).

The scalar curvature $R$ and the co-co-variant Einstein tensor $\boldsymbol{G} \equiv \mathbf{G}_{\text {.. }}$

$$
R:=\operatorname{tr}\left(\boldsymbol{R i c ~}^{-1}\right), \quad \text { G.. := Ric }-\frac{1}{2} R \mathbf{g}
$$

have dimensions

$$
\begin{gathered}
\operatorname{dim}(R)=\operatorname{dim}(\mathbf{g})^{-1} \equiv\left\{\begin{array}{ll}
\mathrm{L}^{-2} & \text { if } \operatorname{dim}(\mathbf{g}):=\mathrm{L}^{2} \\
\mathrm{~T}^{-2} & \text { if } \operatorname{dim}(\mathbf{g}):=\mathrm{T}^{2}
\end{array},\right. \\
\operatorname{dim}\left(\boldsymbol{G}_{. .}\right)=1,
\end{gathered}
$$

that is, the twice covariant Einstein tensor is dimensionless, independently of the dimension of the metric tensor.

\section{Stress-energy-momentum tensor}

Also in the case of the stress-energy-momentum tensor the literature offers two main choices of intrinsic dimension, independent of the choices

43 Choquet-Bruhat et al. 1996 § V.B.2. 
for the metric tensor discussed in the previous section. Moreover, there seems to be no consensus yet on what the primitive variance type of the stress-energy-momentum tensor should be. Its operational meaning is still surrounded by some mystery. Let's try to find its dimension and variance type through a heuristic approach, which will also show the usefulness of intrinsic dimensional analysis on differential manifolds.

The stress-energy-momentum tensor for a material continuum at a spacetime event embodies the volumic energy (comprising rest energy, internal energy, kinetic energy) $\epsilon$, areic energy flux $q_{r}$ (comprising convected volumic energy and heating), volumic momentum $p_{r}$, and stress $\sigma_{s r}$ (considered as compressive rather than tensile, and including convected volumic momentum) of the material at that event. ${ }^{44}$ Here the vertical position of the indices $r, s \in\{x, y, z\}$ does not denote any variance type. These quantities are measured by an inertial observer at that event, using a system of one timelike and three spacelike coordinates $(t, x, y, z)$. If these coordinates have dimensions $(T, L, L, L)$, then the dimensions of the quantities are

$$
\begin{aligned}
\operatorname{dim}(\epsilon) & =\mathrm{ML}^{-1} \mathrm{~T}^{-2} \equiv \mathrm{EL}^{-3}, \quad \operatorname{dim}\left(q_{r}\right)=\mathrm{MT}^{-3} \equiv \mathrm{EL}^{-2} \mathrm{~T}^{-1}, \\
\operatorname{dim}\left(p_{r}\right) & =\mathrm{ML}^{-2} \mathrm{~T}^{-1} \equiv \mathrm{EL}^{-4} \mathrm{~T}, \\
\operatorname{dim}\left(\sigma_{r s}\right) & =\mathrm{ML}^{-1} \mathrm{~T}^{-2} \equiv \mathrm{EL}^{-3} .
\end{aligned}
$$

Suppose we want to construct a tensor $\mathcal{T}$ having these 16 independent quantities as components. What should its variance type and its intrinsic dimension be? I am not assuming the symmetry of this tensor as an apriori kinematic property, leaving it instead as a dynamical law enforced by the Einstein equations; in fact this symmetry only needs to hold for the sum of the stress-energy-momentum tensors from all kinds of matter.

Since we have 16 components, this tensor should belong to the tensor product of two tangent spaces, each spanned by four basis elements. There are four such spaces: vectors, covectors, 3-vectors, and 3-covectors. Let's use shorthands such as $\partial_{t z y}:=\partial_{t} \wedge \partial_{z} \wedge \partial_{y}$ and $\mathrm{d} t z y:=\mathrm{d} t \wedge \mathrm{d} z \wedge \mathrm{d} y$. These four spaces then have the following coordinate-induced bases and

\footnotetext{
44 For the "volumic" and "areic" terminology see Iso 2009 § A.6.
} 
corresponding dimensions:

$$
\begin{array}{ll}
\left(\partial_{t}, \partial_{x}, \partial_{y}, \partial_{z}\right): & \left(\mathrm{T}^{-1}, \mathrm{~L}^{-1}, \mathrm{~L}^{-1}, \mathrm{~L}^{-1}\right), \\
(\mathrm{d} t, \mathrm{~d} x, \mathrm{~d} y, \mathrm{~d} z): & (\mathrm{T}, \mathrm{L}, \mathrm{L}, \mathrm{L}), \\
\left(\partial_{x y z}, \partial_{t z y}, \partial_{t x z}, \partial_{t y x}\right): & \left(\mathrm{L}^{-3}, \mathrm{~L}^{-2} \mathrm{~T}^{-1}, \mathrm{~L}^{-2} \mathrm{~T}^{-1}, \mathrm{~L}^{-2} \mathrm{~T}^{-1}\right), \\
(\mathrm{d} x y z, \mathrm{~d} t z y, \mathrm{~d} t x z, \mathrm{~d} t y x): & \left(\mathrm{L}^{3}, \mathrm{~L}^{2} \mathrm{~T}, \mathrm{~L}^{2} \mathrm{~T}, \mathrm{~L}^{2} \mathrm{~T}\right),
\end{array}
$$

where the orderings are chosen to minimize the minus signs appearing from inner products with a volume element. There are therefore $4 \times 4$ possible tensor-product spaces, each constructed by the product of two of the four spaces above; and thus sixteen possible alternatives to represent our stress-energy-momentum tensor. Volumic energy is intuitively associated with the purely timelike component of this tensor, stress with the purely spacelike components, and areic energy flux and volumic momentum with the mixed timelike-spacelike components.

Consider the following first alternative, obtained from the tensor product of the space (61a) with itself; omit $y$ - and $z$-terms for brevity:

$$
\mathcal{T} \stackrel{?}{=} \epsilon \partial_{t} \otimes \partial_{t}+q_{x} \partial_{t} \otimes \partial_{x}+p_{x} \partial_{x} \otimes \partial_{t}+\sigma_{x x} \partial_{x} \otimes \partial_{x}+\cdots
$$

The first and third summands of this expression have incompatible intrinsic dimensions $\mathrm{EL}^{-3} \mathrm{~T}^{-2}$ and $\mathrm{EL}^{-5}$. This alternative is therefore rejected because dimensionally inconsistent. Similar dimensional analyses on the remaining fifteen alternatives show that only four are dimensionally consistent:

$$
\begin{gathered}
\mathcal{T}_{\bullet}=-\epsilon \mathrm{d} t \otimes \partial_{t}-q_{x} \mathrm{~d} t \otimes \partial_{x}+p_{x} \mathrm{~d} x \otimes \partial_{t}+\sigma_{x x} \mathrm{~d} x \otimes \partial_{x}+\cdots \\
\mathcal{T}_{\cdot|\cdots .|}=-\epsilon \mathrm{d} t \otimes \mathrm{d} x y z-q_{x} \mathrm{~d} t \otimes \mathrm{d} t z y+ \\
p_{x} \mathrm{~d} x \otimes \mathrm{d} x y z+\sigma_{x x} \mathrm{~d} x \otimes \mathrm{d} t z y+\cdots \\
\mathcal{T}^{|\cdots \cdot| \cdot}=-\epsilon \partial_{x y z} \otimes \partial_{t}-q_{x} \partial_{x y z} \otimes \partial_{x}+ \\
p_{x} \partial_{t z y} \otimes \partial_{t}+\sigma_{x x} \partial_{t z y} \otimes \partial_{x}+\cdots
\end{gathered}
$$

$$
\begin{array}{r}
\left.\mathcal{T}^{|\cdots \cdot|}\right|_{|\cdots|}=-\epsilon \partial_{x y z} \otimes \mathrm{d} x y z-q_{x} \partial_{x y z} \otimes \mathrm{d} t z y+ \\
p_{x} \partial_{t z y} \otimes \mathrm{d} x y z+\sigma_{x x} \partial_{t z y} \otimes \mathrm{d} t z y+\cdots
\end{array}
$$

where the particular signs of the components, which are not determined by dimensional analysis, will be motivated later. Our analysis of the 
intrinsic dimensions therefore restricts the stress-energy-momentum tensor to be one of the four alternatives above, with their kinds of orientation, straight or twisted, still undetermined. Note that if we had conflated time and length dimensions in eqs (60) and (61) by introducing $c$ factors, then dimensional analysis wouldn't have led to any restrictions: all sixteen alternatives would have been dimensionally consistent. This shows the usefulness - at times at least - to keep dimensions well distinct.

To further restrict the possibilities let's consider three additional and interrelated heuristic arguments.

First, the notions of volumic energy and momentum, areic energy flux, and stress imply some kind of integration over three-dimensional spacelike or timelike regions. Such integration needs a 3-form and thus excludes alternatives (62a) and (62c).

Second, the total energy measured within a topologically specified three-dimensional spatial region of an observer's orthogonal hyperplane is considered to be independent of the extent of that region, whether the extent is reckoned by a physical metric or by arbitrary coordinate intervals. The volumic energy therefore does depend on the extent of the region and must change accordingly. Similar arguments hold for the surface energy flux. Only the second tensor alternative (62b) above is consistent with these requirements.

This extent-scaling argument also suggests that the volumicmomentum part $p_{x} \mathrm{~d} x \otimes \mathrm{d} x y z$ should not be interpreted as an areic mass flux $\mathrm{ML}^{-2} \mathrm{~T}^{-1}$, that is, as something that needs to be integrated over a surface: the basis element $\mathrm{d} x \otimes \mathrm{d} x y z$ indicates that integration should happen over a volume instead: $\operatorname{dim}\left(p_{r}\right)=\left(\mathrm{MLT}^{-1}\right) \mathrm{L}^{-3}$. A similar situation occurs for the stress part, which should be interpreted as an areic momentum flux: $\operatorname{dim}\left(\sigma_{r s}\right)=\left(\mathrm{MLT}^{-1}\right) \mathrm{L}^{-2} \mathrm{~T}^{-1}$. It is worth noting scaling reinterpretations of this kind occurs, even for more components, in all other fifteen alternatives for the stress-energy-momentum tensor. Intrinsic dimensional analysis alone thus suggests differences between mass flux and momentum.

Third, the value of the energy density should not change under a change in the orientation of the spacelike coordinates. The 3-covector slot in alternative (62b) should therefore have a twisted orientation. This means that the 3-form $\mathrm{d} x y z$ actually has an inner orientation in the positive $t$ direction, the 3 -form $\mathrm{d} t z y$ in the positive $x$ direction, and so 
on ${ }^{45}$.

A heuristic application of intrinsic dimensional analysis combined with integration, scaling, and orientation arguments thus tells us that the stress-energy-momentum tensor has variance type $\mathcal{T}_{.|. . .|}$, that is, it's a covector-valued 3-covector, or a four-times-covariant tensor completely antisymmetric in three slots. The 3-covector part has a twisted orientation. This tensor has the dimension of an action:

$$
\operatorname{dim}\left(\mathcal{T}_{\bullet|\ldots| \mid}\right)=\mathrm{ET} \equiv \mathrm{ML}^{2} \mathrm{~T}^{-1}
$$

This result agrees with the stress-energy-momentum tensor that appears for example in Einstein's original work ${ }^{46}$, other early works ${ }^{47}$, and more recent works ${ }^{48}$; and also in Truesdell \& Toupin ${ }^{49}$, who try to find an expression universally valid in Newtonian, Lorentzian, and generalrelativistic mechanics. The commonly encountered versions of this tensor with only two slots are discussed below. Note that some of these works use a once covariant and once contravariant "V(olume)-tensor" or "tensor density", which has variance type $\mathcal{T} \cdot|\ldots .$.$| . Such an object,$ however, is geometrically equivalent to the variance type $\mathcal{T}_{\bullet|. . .|}$; their independent components have the same transformation law under changes of coordinates ${ }^{50}$ (this is why I chose a calligraphic letter to denote this tensor).

The signs of the components of $\mathcal{T}$ depend on the signature of the metric $g$. If the latter has signature $(-,+,+,+)$, then the energy components have negative sign, as in eq. (62b). If the metric has opposite sign, that is, signature $(+,-,-,-)$, then $\mathcal{T}$ has opposite sign to eq. (62b) as well, and its momentum components are negative instead.

The literature cited above arrive at this kind of stress-energymomentum tensor through inductive generalization, often via electromagnetic theory, of the stress tensor of Newtonian mechanics; or from principles of virtual work; or from variational principles with an

45 To visualize this cf. Schouten 1989 Fig. 6 and table in § II.8 p. 31; and Burke 1987 Fig. 22.10. $\quad 46$ Einstein 1914 § C.9, discussion before eq. (42a). $\quad 47$ e.g. Pauli 1958 § IV.54; Cartan 1923 § 13; Brillouin 1924 § $7 . \quad 48$ Fokker 1965 § VIII.3; Misner et al. 1973 ch. 14 Exercise 14.18, ch. 15, § 21.3; Hehl \& McCrea 1986; Gotay \& Marsden 1992; Gronwald \& Hehl 1997; Castrillón López et al. 2008; 2009; see also Segev \& Rodnay 1999; Kanso et al. 2007 for similar conclusions in general manifolds and in Newtonian mechanics.

49 Truesdell \& Toupin 1960 § F.IV.288. $\quad{ }^{\mathbf{5 0}}$ Schouten 1989 § II.8 p. 30. 
action Lagrangean ${ }^{51}$, from which it easily follows that this tensor should have the intrinsic dimension of an action; or from combinations of these approaches. The operational meaning of this tensor is therefore still unclear in my opinion.

In the presence of a metric tensor we can of course obtain stressenergy-momentum tensors of different variance types by means of inner products with the proper volume element and its inverse, and by raising and lowering indices. But the question of the operational meaning and primitive variance type of this tensor are important, for example, in field theories not based on a metric, or for the formulation of constitutive equations ${ }^{52}$. Extensive investigations were made by Gotay et al. ${ }^{53}$; and by Segev ${ }^{54}$, who interprets the stress-energy-momentum tensor as a linear map from the four-dimensional flux of a conserved quantity, such as charge or baryonic number, to that of energy. Since such fluxes are represented by 3-forms, he arrives at the fourth alternative (62d) above: the 3-vector part of $\mathcal{T}^{1 \cdots 1}{ }_{|\cdots|}$ can be contracted with a 3-form, yielding another 3-form. This interesting interpretation doesn't seem to work out dimensionally, however. The intrinsic dimension of the four-dimensional charge flux, for example, is charge itself, $Q \equiv I T$, and similarly for the energy flux. The tensor $\mathcal{T}^{|\cdots|}|\ldots|$ should then have intrinsic dimension $\mathrm{EQ}^{-1}$ according to the results of $\S 6$. The dimension of charge would then have to appear in Einstein's constant $\kappa$ (see § 11), because it cannot be eliminated by using the metric tensor or the proper volume element to obtain alternative variance types.

Some authors ${ }^{55}$ conceive the stress-energy-momentum tensor in terms of mass rather than energy (Fock ${ }^{56}$ calls it the "mass tensor"), and therefore assign to its covector-valued 3-covector form $\mathcal{T}_{.|\ldots .|}$ the dimension of mass-time, that is, an action divided by squared velocity:

$$
\operatorname{dim}\left(\mathcal{T}_{\bullet|\ldots| \mid}\right)=M T \equiv E L^{-2} T^{3} .
$$

With this intrinsic dimension, however, not all components of the stressenergy-momentum tensor have intuitive meanings and dimensions when coordinates with dimensions $(T, L, L, L)$ are used. The two choices (63), (64) differ by a factor $c^{2}$.

\footnotetext{
51 cf. also Hilbert 1915; 1917; Hawking \& Ellis 1994 § 3.3. $\quad 52$ cf. Truesdell \& Toupin 1960 ch. G; Marsden \& Hughes 1994; Gotay \& Marsden 1992. 53 Gotay et al. 1998; 2004; Gotay \& Marsden 2006. 54 Segev 2002; see also Segev 2000. $\quad 55$ e.g. Fock 1964 § V.55; McVittie 1965 § 4.1; Adler et al. 1975 § 10.1. $\quad 56$ Fock 1964 § II.31.
} 
The two dimensional choices for the metric, eqs (42) and (44), and for the stress-energy-momentum tensor, eqs (63) and (64), appear in all four combinations in the literature. For example, $\operatorname{dim}(\mathbf{g})=\mathrm{T}^{2}$ and $\operatorname{dim}(\mathcal{T})=$ ET is used by Synge ${ }^{57} ; \operatorname{dim}(\boldsymbol{g})=\mathrm{L}^{2}$ and $\operatorname{dim}(\mathcal{T})=$ MT is used by Fock ${ }^{58}$ and Adler et al. ${ }^{59} ; \operatorname{dim}(\boldsymbol{g})=\mathrm{T}^{2}$ and $\operatorname{dim}(\mathcal{T})=\mathrm{MT}$ is used by McVittie $^{60}$ and possibly Kilmister ${ }^{61}$. Most other works use $\operatorname{dim}(\mathbf{g})=\mathrm{L}^{2}$ and $\operatorname{dim}(\mathcal{T})=$ ET. These combinations lead to three possible values for Einstein's constant, discussed in the next section.

To obtain a twice covariant tensor to be used in the Einstein equations, we first take the inner product of the inverse proper volume element with the 3-covector (that is, antisymmetric) part of $\mathcal{T}_{.|. . .|}$, obtaining a co-contravariant tensor. Then we lower the new contravariant slot by means of the metric tensor. The combined operation yields

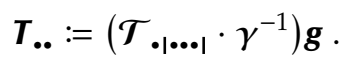

According to this definition and eqs (64), (42), (44), (50), the co-covariant tensor $\boldsymbol{T}$ has three possible intrinsic dimensions, depending on the choices of dimensions of $\boldsymbol{g}$ and $\mathcal{T}$ :

$$
\begin{aligned}
& \operatorname{dim}\left(\boldsymbol{T}_{\text {.. }}\right)=\operatorname{dim}\left(\mathcal{T}_{.|\boldsymbol{*} \boldsymbol{|}|}\right) \operatorname{dim}(\boldsymbol{g}) \operatorname{dim}(\boldsymbol{\gamma})^{-1}= \\
& \begin{cases}M L T^{-2} \equiv E L^{-1} & \text { if } \operatorname{dim}(\mathbf{g}):=\mathrm{L}^{2}, \operatorname{dim}(\mathcal{T}):=\mathrm{ET} \\
\mathrm{ML}^{-1} \equiv E L^{-3} \mathrm{~T}^{2} & \text { if }\left\{\begin{array}{l}
\operatorname{dim}(\boldsymbol{g}):=\mathrm{T}^{2}, \operatorname{dim}(\mathcal{T}):=\mathrm{ET} \quad \text { or } \\
\operatorname{dim}(\boldsymbol{g}):=\mathrm{L}^{2}, \operatorname{dim}(\mathcal{T}):=\mathrm{MT}
\end{array}\right. \\
\mathrm{ML}^{-3} \mathrm{~T}^{2} \equiv E L^{-5} \mathrm{~T}^{4} & \text { if } \operatorname{dim}(\boldsymbol{g}):=\mathrm{T}^{2}, \operatorname{dim}(\mathcal{T}):=\mathrm{MT}\end{cases}
\end{aligned}
$$

All three possibilities, which differ by factors $c^{2}$, appear in the literature: see the works cited after eq. (63) concerning the combinations of dimensions for metric and stress-energy-momentum.

It may be useful to write the coordinate expressions of the tensors $\boldsymbol{T}_{\text {.. }}$ and $\boldsymbol{T}^{\boldsymbol{\bullet}}$ obtained from $\mathcal{T}_{\text {.|... }}$ of eq. (62b), in the common case with

57 Synge 1960a §§ IV.4-5. $\quad$ 58 Fock 1964 §§ V.54-55. $\quad \quad{ }^{\mathbf{5 9}}$ Adler et al. $1975 \S$ 10.1.

$\mathbf{6 0}$ McVittie $1965 \S 4.1 . \quad 61$ Kilmister 1973 chs II-III, he seems to shift to natural units at some point. 
$\operatorname{dim}(\mathbf{g}):=\mathrm{L}^{2}, \operatorname{dim}(\mathcal{T}):=\mathrm{ET}$, and coordinate system with diagonal metric $\mathbf{g}= \pm\left(-c^{2} \mathrm{~d} t \otimes \mathrm{d} t+\mathrm{d} x \otimes \mathrm{d} x+\cdots\right)$, commonly encountered in the literature:

$$
\begin{aligned}
& \boldsymbol{T}_{. \cdot}=c^{2} \epsilon \mathrm{d} t \otimes \mathrm{d} t-q_{x} \mathrm{~d} t \otimes \mathrm{d} x-c^{2} p_{x} \mathrm{~d} x \otimes \mathrm{d} t+\sigma_{x x} \mathrm{~d} x \otimes \mathrm{d} x+\cdots \\
& \boldsymbol{T}^{\cdot \bullet}=\frac{1}{c^{2}} \epsilon \partial_{t} \otimes \partial_{t}+\frac{1}{c^{2}} q_{x} \partial_{t} \otimes \partial_{x}+p_{x} \partial_{x} \otimes \partial_{t}+\sigma_{x x} \partial_{x} \otimes \partial_{x}+\cdots
\end{aligned}
$$

where the $c$ factors can be freely interpreted as part either of the components or of the $t$ coordinate. Remember that the stress is here considered as compressive rather than tensile, and that the Einstein equations require in particular that $q_{x}=c^{2} p_{x}$ and so on (heat flux carries momentum ${ }^{62}$ ).

\section{The constant in the Einstein equations}

We finally arrive at the Einstein equations,

$$
\boldsymbol{G}=\kappa \boldsymbol{T}
$$

sometimes seen with a minus sign, depending on the signature of the metric and of alternative definitions of the curvature tensors ${ }^{63} . \kappa$ is Einstein's constant.

The equations above are considered in their co-co-variant form. This form is convenient because the left side is then dimensionless (its intrinsic dimension is 1), independently of the dimension of the metric tensor, as explained in $\S 9.4$, eq. (59). We therefore find that the equality

$$
\operatorname{dim}(\kappa)=\operatorname{dim}\left(\boldsymbol{T}_{\text {.. }}\right)^{-1}
$$

must always hold, for all choices of dimensions for the metric and stressenergy-momentum tensors. Combining this equation with the results for $\boldsymbol{T}$, eq. (66), we find three possible conventions:

$$
\operatorname{dim}(\kappa)=
$$

$$
\begin{cases}M^{-1} L^{-1} T^{2} \equiv E^{-1} L & \text { if } \operatorname{dim}(\mathbf{g}):=\mathrm{L}^{2}, \operatorname{dim}(\mathcal{T}):=E T \\
M^{-1} \mathrm{~L} \equiv E^{-1} \mathrm{~L}^{3} \mathrm{~T}^{-2} & \text { if }\left\{\begin{array}{l}
\operatorname{dim}(\boldsymbol{g}):=\mathrm{T}^{2}, \operatorname{dim}(\mathcal{T}):=\mathrm{ET} \quad \text { or } \\
\operatorname{dim}(\boldsymbol{g}):=\mathrm{L}^{2}, \operatorname{dim}(\mathcal{T}):=M T
\end{array}\right. \\
M^{-1} \mathrm{~L}^{3} \mathrm{~T}^{-2} \equiv E^{-1} \mathrm{~L}^{5} \mathrm{~T}^{-4} & \text { if } \operatorname{dim}(\boldsymbol{g}):=\mathrm{T}^{2}, \operatorname{dim}(\mathcal{T}):=M T\end{cases}
$$

62 Eckart 1940 p. $923 . \quad 63$ see the Table of sign conventions on the final pages of Misner et al. 1973. 
Einstein's constant $\kappa$ can therefore be obtained from Newton's gravitational constant $\operatorname{dim}(G)=\mathrm{M}^{-1} \mathrm{~L}^{3} \mathrm{~T}^{-2}$ (this is not the Einstein tensor $\boldsymbol{G}$ !) and the speed of light $\operatorname{dim}(c)=\mathrm{LT}^{-1}$ only in the following three ways, with the $8 \pi$ factor coming from the Newtonian limit:

$$
\kappa= \begin{cases}8 \pi G / c^{4} & \text { if } \operatorname{dim}(\mathbf{g}):=\mathrm{L}^{2}, \operatorname{dim}(\mathcal{T}):=\mathrm{ET} \\
8 \pi G / c^{2} & \text { if }\left\{\begin{array}{l}
\operatorname{dim}(\mathbf{g}):=\mathrm{T}^{2}, \operatorname{dim}(\mathcal{T}):=\mathrm{ET} \quad \text { or } \\
\operatorname{dim}(\mathbf{g}):=\mathrm{L}^{2}, \operatorname{dim}(\mathcal{T}):=\mathrm{MT}
\end{array}\right. \\
8 \pi G & \text { if } \operatorname{dim}(\boldsymbol{g}):=\mathrm{T}^{2}, \operatorname{dim}(\mathcal{T}):=\mathrm{MT}\end{cases}
$$

As we saw in the discussion of the literature cited in $\S 10$, the first convention is the most common. The second convention appears for example in Fock ${ }^{64}$ and Adler et al. ${ }^{65}$. The third, interesting convention would appear in McVittie if he didn't cheat a factor $1 / c^{2}$ into $\kappa$ by writing the Einstein equations ${ }^{66}$ as " $\boldsymbol{G}=\kappa c^{2} \boldsymbol{T}$ ".

\section{Summary and conclusions}

We have seen that dimensional analysis, with its familiar rules, can be seamlessly performed in Lorentzian and general relativity and in differential geometry if we adopt the coordinate-free approach typical of modern texts. In this approach each tensor has an intrinsic dimension, a notion introduced by Schouten and Dorgelo. This dimension doesn't depend on the dimensions of the coordinates, and is determined by the physical and operational meaning of the tensor. It is therefore generally more profitable to focus on the intrinsic dimension of a tensor rather than on the dimensions of its components. The dimension of each specific component is easily found by formula (12): it's the product of the intrinsic dimension by the dimension of the $i$ th coordinate function for each contravariant index $i$, by the inverse of the dimension of the $j$ th coordinate function for each covariant index $j$. Intrinsic dimensional analysis seems to rest on two main conventions: the tensor product and the action of covectors on vectors behave analogously to usual multiplication for the purposes of dimensional analysis. Alternative, equivalent sets of conventions could perhaps be considered.

${ }^{64}$ Fock $1964 \S 55$ eqs (55.15) and (52.06).

66 McVittie 1965 § 4.2 eq. (4.107).

65 Adler et al. 1975 § 10.5 eq. (10.98). 
We have also seen that intrinsic dimensional analysis can help us determine or at least constrain the variance type of candidate tensors, as exemplified with the stress-energy-momentum tensor in $\S 10$. We found or re-derived some essential results for general relativity, in particular that the Riemann $\boldsymbol{R i e}_{{ }_{. \mid .0 .}}$, Ricci Ric.., and twice covariant Einstein $\boldsymbol{G}$.. curvature tensors are dimensionless. These results could be of importance for current research involving scales and conformal factors ${ }^{67}$. We also discussed the operational reasons behind two common choices of dimensions for the metric and stress-energy-momentum tensors.

Since the dimensions of the components are usually different from the intrinsic dimension and depend on the coordinates, I recommend to avoid statements such as "the tensor $A_{i}^{j k}$ has dimension X", which leave

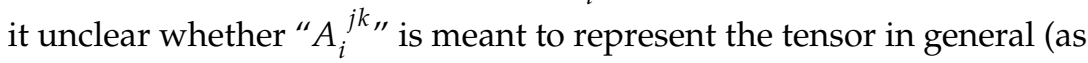
in Penrose \& Rindler's notation), or to represent its set of components, or to represent only a specific component.

For the dimensional analysis of tensorial objects in electrodynamics, which wasn't discussed in this note, see for example Truesdell \& Toupin ${ }^{68}$ and Hehl \& Obukhov ${ }^{69}$.

Dimensional analysis remains a controversial, obscure, but fascinating subject still today, 60 years from Truesdell \& Toupin's remark quoted in the Introduction. For an overview of some recent and creative approaches to it, going beyond Bridgman's text ${ }^{70}$ (whose point of view is in many respects at variance with modern developments: see the following references), I recommend for example the works by Mari et al. ${ }^{71}$, Domotor and Batitsky ${ }^{72}, \mathrm{Kitano}^{73}$, the extensive analysis by Dybkaer ${ }^{74}$, the historical review by de Boer ${ }^{75}$, and references therein.

\section{Thanks}

... to (chronologically) Mariano Cadoni, Ingemar Bengtsson, Iván Davidovich, Claudia Battistin for valuable comments on previous drafts. To the staff of the NTNU library for their always prompt support. To

67 e.g. Röhr \& Uggla 2005; Cadoni \& Tuveri 2019. $\quad 68$ Truesdell \& Toupin 1960

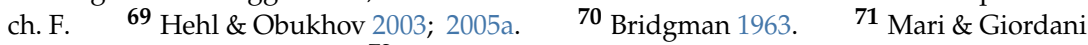
2012; Frigerio et al. 2010. 72 Domotor 2017; Domotor \& Batitsky 2016; Domotor 2012.

73 Kitano 2013. $\quad 74$ Dybkær 2010. $\quad{ }^{75}$ De Boer 1995. 
Mari, Miri, Emma for continuous encouragement and affection, and to Buster Keaton and Saitama for filling life with awe and inspiration. To the developers and maintainers of LTEX, Emacs, AUCTEX, Open Science Framework, R, Python, Inkscape, Sci-Hub for making a free and impartial scientific exchange possible.

Note: this paper was rejected by arXiv without any kind of scientific justification.

This work was financially supported partly by the Kavli Foundation and the Centre of Excellence scheme of the Research Council of Norway (Roudi group), partly by the Trond Mohn Research Foundation, grant number BFS2018TMT07.

\section{Bibliography}

("de $X$ " is listed under D, "van $X$ " under V, and so on, regardless of national conventions.)

Abraham, R., Marsden, J. E., Ratiu, T. (1988): Manifolds, Tensor Analysis, and Applications, 2nd ed. (Springer, New York). First publ. 1983. Dor:10 . 1007/978 - 1- 4612 - 1029 - 0.

Adler, R., Bazin, M., Schiffer, M. (1975): Introduction to General Relativity, 2nd ed. (McGrawHill, Tokyo). First publ. 1965.

Alcubierre, M. (2008): Introduction to $3+1$ Numerical Relativity. (Oxford University Press, Oxford).

BIPM (Bureau international des poids et mesures) (1983): $17^{e}$ conférence générale des poids et mesures (CGPM). (BIPM, Sèvres). https://www. bipm. org/en/worldwide-metrology/cg $\mathrm{pm} /$ resolutions. html\#comptes - rendus.

Boothby, W. M. (2003): An Introduction to Differentiable Manifolds and Riemannian Geometry, rev. 2nd ed. (Academic Press, Orlando, USA). First publ. 1975.

Bossavit, A. (1991): Differential Geometry: for the student of numerical methods in electromagnetism. https://Www. researchgate. net/publication/200018385 Differential Geome try_for_the_student_of_numerical_methods_in_Electromagnetism.

Bressan, A. (1978): Relativistic Theories of Materials. (Springer, Berlin). DOI:10 . 1007/978- 3 - $642-81120-3$.

Bridgman, P. W. (1958): The Logic of Modern Physics, 8th pr. (Macmillan, New York). First publ. 1927.

- (1963): Dimensional Analysis, rev. ed. (Yale University Press, New Haven). First publ. 1922. http://archive.org/details/dimensionalanalyoobridrich.

Brillouin, L. (1924): Les lois de l'élasticité en coordonnées quelconques. Proc. Int. Congress Mathematicians 1924-II, 73-97.

Burke, W. L. (1980): Spacetime, Geometry, Cosmology. (University Science Books, Mill Valley, USA).

- (1983): Manifestly parity invariant electromagnetic theory and twisted tensors. J. Math. Phys. 241 , 65-69. DOI:10.1063/1.525603.

- (1987): Applied Differential Geometry, repr. (Cambridge University Press, Cambridge). DOI:10. 1017/CB09781139171786. First publ. 1985. 
Burke, W. L. (1995): Div, Grad, Curl Are Dead. http: //people. ucsc. edu/ rmont/papers /Burke_DivgradCurl.pdf. See also http://www. ucolick.org/ burke/.

Cadoni, M., Tuveri, M. (2019): Galactic dynamics and long-range quantum gravity. Phys. Rev. D 100², 024029. DOI:10.1103/PhysRevD. 100.024029.

Cartan, É. (1923): Sur les variétés à connexion affine et la théorie de la relativité généralisée (première partie). Annales scientifiques de l’École Normale Supérieure 40, 325-412. DOI:10.24033/asens . 751. See also Cartan (1924; 1925); transl. in Cartan (1986).

- (1924): Sur les variétés à connexion affine et la théorie de la relativité généralisée (première partie) (Suite). Annales scientifiques de l'École Normale Supérieure 41, 1-25. DOI:10. 2 4033/asens . 753. See also Cartan (1923; 1925); transl. in Cartan (1986).

- (1925): Sur les variétés à connexion affine et la théorie de la relativité généralisée (deuxième partie). Annales scientifiques de l'École Normale Supérieure 42, 17-88. DOr:10. 24033 /asens . 761. See also Cartan (1923; 1924); transl. in Cartan (1986).

- (1986): On Manifolds with an Affine Connection and the Theory of General Relativity. (Bibliopolis, Napoli). Transl. of Cartan (1923; 1924; 1925) by Anne Magnon and Abhay Ashtekar.

Castrillón López, M., Gotay, M. J., Marsden, J. E. (2008): Parametrization and stress-energymomentum tensors in metric field theories. J. Phys. A 41 ${ }^{34}, 344002$. DOI:10. 1088/1751- 81 $13 / 41 / 34 / 344002$.

- (2009): Concatenating variational principles and the kinetic stress-energy-momentum tensor. In: Krupková, Saunders (2009): p. 7:117-128.

Choquet-Bruhat, Y., DeWitt-Morette, C., Dillard-Bleick, M. (1996): Analysis, Manifolds and Physics. Part I: Basics, rev. ed. (Elsevier, Amsterdam). First publ. 1977.

Curtis, W. D., Miller, F. R. (1985): Differential Manifolds and Theoretical Physics. (Academic Press, Orlando, USA).

de Boer, J. (1995): On the history of quantity calculus and the International System. Metrologia 316 , 405-429. DOI:10. 1088/0026 - 1394/31/6/001.

de Rham, G. (1984): Differentiable Manifolds: Forms, Currents, Harmonic Forms. (Springer, Berlin). Transl. by F. R. Smith. First publ. in French 1955. Dor:10. 1007/978 - 3- 642 - 617 52- 2.

Deschamps, G. A. (1970): Exterior differential forms. In: Deschamps, de Jager, John, Lions, Moisseev, Sommer, Tihonov, Tikhomirov, et al. (1970): p. III:111-161.

- (1981): Electromagnetics and differential forms. Proc. IEEE 696 6 676-696. DOI:10.1109 /PROC. 1981. 12048, https://WWW-liphy.ujf-grenoble.fr/pagesperso/bahram/bibl io/Deschamps1981_dif_forms.pdf.

Deschamps, G. A., de Jager, E. M., John, F., Lions, J. L., Moisseev, N., Sommer, F., Tihonov, A. N., Tikhomirov, V., et al. (1970): Mathematics applied to physics. (Springer, Berlin). Ed. by É. Roubine.

Domotor, Z. (2012): Algebraic frameworks for measurement in the natural sciences. Meas. Sci. Rev. 126 ${ }^{6}$ 213-233. DOI:10.2478/v10048-012-0032- 7.

- (2017): Torsor theory of physical quantities and their measurement. Meas. Sci. Rev. 174, 152-177. DOI:10. 1515/ms r- 2017-0019.

Domotor, Z., Batitsky, V. (2016): An algebraic approach to unital quantities and their measurement. Meas. Sci. Rev. 16 ${ }^{3}, 103-126$. DOI:10. 1515/ms r- 2016 - 0014.

Dorgelo, H. B., Schouten, J. A. (1946): On unities and dimensions. [I, II, III]. Verh. Kon. Akad. Wetensch. Amsterdam 492, 3, 4, 123-131, 282-291, 393-403. 
Dybkær, R. (2010): An Ontology on Property: For Physical, Chemical and Biological Systems, 2nd ed. (IUPAC, Research Triangle Park, USA). First publ. 2004. Dor:10. 1351/978 - 87 - 9 90010 - 1- 9 .

Eckart, C. (1940): The thermodynamics of irreversible processes. III. Relativistic theory of the simple fluid. Phys. Rev. 58 ${ }^{10}, 919-924$. Dor:10.1103/PhysRev . 58.919.

Einstein, A. (1914): Die formale Grundlage der allgemeinen Relativitätstheorie. Sitzungsber.

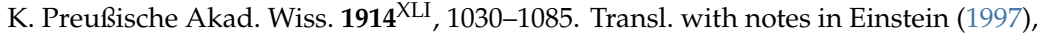
Doc. 9 pp. $30-84$.

- (1996): The Collected Papers of Albert Einstein. Vol. 6: The Berlin Years: Writings, 1914-1917. (Princeton University Press, Princeton). Ed. by A. J. Kox, Martin J. Klein, Robert Schulmann. Transl. in Einstein (1997).

- (1997): The Collected Papers of Albert Einstein. Vol. 6: The Berlin Years: Writings, 1914-1917. English translation of selected texts. (Princeton University Press, Princeton). Transl. of Einstein (1996) by Alfred Engel and Engelbert Schucking.

Fecko, M. (2006): Differential Geometry and Lie Groups for Physicists. (Cambridge University Press, Cambridge). Dor:10.1017/CB09780511755590.

Flügge, S., ed. (1960): Handbuch der Physik: Band III/1: Prinzipien der klassischen Mechanik und Feldtheorie [Encyclopedia of Physics: Vol. III/1: Principles of Classical Mechanics and Field Theory]. (Springer, Berlin). Dor:10. 1007/978- 3-642-45943-6.

Fock [Fok], V. A. (1964): The Theory of Space, Time and Gravitation, 2nd rev. ed. (Pergamon, Oxford). Transl. by N. Kemmer. Dor:10. 1016/C2013- - - 05319- 4. First publ. in Russian 1955.

Fokker, A. D. (1965): Time and Space, Weight and Inertia: A chronogeometrical introduction to Einstein's theory. (Pergamon, Oxford). Transl. by D. Bijl, translation ed. by D. Field. First publ. in Dutch 1960.

Frankel, T. (1979): Gravitational Curvature: An Introduction to Einstein's Theory. (W. H. Freeman and Company, San Francisco).

Frigerio, A., Giordani, A., Mari, L. (2010): Outline of a general model of measurement. Synthese 1752, 123-149. DOI:10. 1007/s11229-009- 9466 - 3.

Giacomo, P. (1984): News from the BIPM. Metrologia 201 ${ }^{1}$ 25-30. DOI:10 . 1088/0026 - 1394/1 7/2/007.

Gotay, M. J., Isenberg, J., Marsden, J. E. (2004): Momentum maps and classical relativistic fields. Part II: Canonical analysis of field theories. arXiv: math - ph/0411032.

Gotay, M. J., Isenberg, J., Marsden, J. E., Montgomery, R. (1998): Momentum maps and classical relativistic fields. Part I: Covariant field theory. arXiv: physics/9801019.

Gotay, M. J., Marsden, J. E. (1992): Stress-energy-momentum tensors and the Belinfante-Rosenfeld formula. Contemp. Math. 132, 367-392. http://www. cds. caltech. edu/ marsden/bib /1992. html.

- (2006): Momentum maps and classical relativistic fields. Part III: Gauge symmetries and initial value constraints. https://www. pims. math. ca/ gotay/.

Gourgoulhon, É. (2012): 3+1 Formalism in General Relativity: Bases of Numerical Relativity. (Springer, Heidelberg). First publ. 2007 as arXiv : gr - qc/0703035. DOI:10 . 1007/978- 3 - 642 - 24525 - 1.

Griffies, S. M. (2004): Fundamentals of Ocean Climate Models. (Princeton University Press, Princeton).

Gronwald, F., Hehl, F. W. (1997): Stress and hyperstress as fundamental concepts in continuum mechanics and in relativistic field theory. arXiv : gr-qc/9701054. 
Hartle, J. B. (2003): Gravity: An Introduction to Einstein's General Relativity. (Addison-Wesley, San Francisco).

Hawking, S. W., Ellis, G. F. R. (1994): The Large Scale Structure of Space-Time, repr. (Cambridge University Press, Cambridge). DoI:10. 1017/CB09780511524646.

Hehl, F. W., McCrea, J. D. (1986): Bianchi identities and the automatic conservation of energymomentum and angular momentum in general-relativistic field theories. Found. Phys. $\mathbf{1 6}^{3}$, 267-293. DOI:10.1007/BF01889477.

Hehl, F. W., Obukhov, Y. N. (2001): How does the electromagnetic field couple to gravity, in particular to metric, nonmetricity, torsion, and curvature? In: Lämmerzahl, Everitt, Hehl (2001): 479-504.

- (2003): Foundations of Classical Electrodynamics: Charge, Flux, and Metric. (Birkhäuser, Boston). DOI:10 . 1007/978- 1- 4612 - 0051- 2.

- (2005a): Dimensions and units in electrodynamics. Gen. Relat. Gravit. 37 ${ }^{4}$, 733-749. Dor:1 $0.1007 / \mathrm{s} 10714-005-0059-2$.

- (2005b): To consider the electromagnetic field as fundamental, and the metric only as a subsidiary field. Found. Phys. 35 ${ }^{12}, 2007-2025$. Dor:10 . 1007/s10701- 005 - 8659 - y.

Hilbert, D. (1915): Die Grundlagen der Physik (Erste Mitteilung). Ges. d. Wiss. Nachrichten (Gött.) Math.-Phys. Kl. 19153 , 395-407. http:// resolver . sub. uni-goettingen. de/p url?GDZPPN002504286. See also Hilbert (1917).

- (1917): Die Grundlagen der Physik (Zweite Mitteilung). Ges. d. Wiss. Nachrichten (Gött.) Math.-Phys. Kl. 1917², 53-76. http : // resolver . sub . uni-goettingen . de / purl ?GDZPPN002504561. See also Hilbert (1915).

ISO (International Organization for Standardization) (2009): ISO 80000-1:2009: Quantities and units 1: General. International Organization for Standardization.

Kanso, E., Arroyo, M., Tong, Y., Yavari, A., Marsden, J. G., Desbrun, M. (2007): On the geometric character of stress in continuum mechanics. Z. Angew. Math. Phys. 58 ${ }^{5}, 843-856$. http: //Www. geometry. caltech. edu/pubs . html, DOI:10. 1007/s00033-007-6141-8.

Kilmister, C. W. (1973): General Theory of Relativity. (Pergamon, Oxford).

Kitano, M. (2013): Mathematical structure of unit systems. J. Math. Phys. 54 ${ }^{5}$, 052901. Dor:10 $.1063 / 1.4802876$.

Krupková, O., Saunders, D., eds. (2009): Variations, Geometry and Physics. (Nova Science, New York).

Lämmerzahl, C., Everitt, F., Hehl, F. W., eds. (2001): Gyros, Clocks, Interferometers. . . Testing Relativistic Gravity in Space. (Springer, Berlin). DoI:10 . 1007/3 - 540- 40988 - 2.

Landau, L. D., Lifshitz [Lifšic], E. M. (1996): The Classical Theory of Fields, corr. repr. of the 4th English ed. (Butterworth-Heinemann, Oxford). Transl. from the 1987 seventh Russian edition by Morton Hamermesh. First publ. 1939. DoI:10 . 1016/C2009- 0 - 14608 $-1$.

Lindell, I. V. (2004): Differential Forms in Electromagnetics. (IEEE Press and Wiley, Piscataway, USA). DOI:10. 1002/0471723096.

Mari, L., Giordani, A. (2012): Quantity and quantity value. Metrologia 496 , 756-764. DOI:10 $.1088 / 0026$ - 1394/49/6/756.

Marsden, J. E., Hughes, T. J. R. (1994): Mathematical Foundations of Elasticity, unabridged corr. republ. (Dover, New York). http://resolver. caltech. edu/CaltechB00K: 1983 .002. First publ. 1983.

Martin, D. (2004): Manifold Theory: Introduction for Mathematical Physicists, corr. repr. (Horwood, Chichester). First publ. 1991. 
Maugin, G. A. (1974): Constitutive equations for heat conduction in general relativity. J. Phys. A $7^{4}, 465-484$. DOI:10 . 1088/0305-4470/7/4/010.

- (1978a): On the covariant equations of the relativistic electrodynamics of continua. I. General equations. J. Math. Phys. 195, $1198-1205$. Dor:10.1063/1.523785.

- (1978b): On the covariant equations of the relativistic electrodynamics of continua. II. Fluids. J. Math. Phys. 195, $1206-1211$. Dor:10.1063/1.523786.

- (1978c): On the covariant equations of the relativistic electrodynamics of continua. III. Elastic solids. J. Math. Phys. 195 ,1212-1219. DoI:10.1063/1.523787.

- (1978d): On the covariant equations of the relativistic electrodynamics of continua. IV. Media with spin. J. Math. Phys. 195, 1220-1226. DOI:10.1063/1.523788.

McVittie, G. C. (1965): General Relativity and Cosmology, 2nd ed. (Chapman \& Hall, London). First publ. 1956.

Misner, C. W., Thorne, K. S., Wheeler, J. A. (1973): Gravitation, repr. (W. H. Freeman and Company, New York). First publ. 1970. https ://archive.org/details/Gravitation 201803.

Muschik, W., von Borzeszkowski, H.-H. (2014): Exploitation of the dissipation inequality in general relativistic continuum thermodynamics. Arch. Appl. Mech. 849, $1517-1531$. DoI:10 . 1007/s00419-014-0866- 6.

Pauli, W. (1958): Theory of Relativity. (Pergamon, London). Transl. by G. Field; with supplementary notes by W. Pauli. First publ. in German 1921.

Penrose, R., Rindler, W. (2003): Spinors and Space-Time. Vol. 1: Two-spinor calculus and relativistic fields, corr. repr. (Cambridge University Press, Cambridge). First publ. 1984. DOI:10. 1017/CB09780511564048.

Porta Mana, P. G. L. (2019a): Answer to "MTW's Gravitation. Factor of contraction of p-vector with p-form". https://math. stackexchange. com/a/3208204/455507.

- (2019b): Affine and convex spaces: blending the analytic and geometric viewpoints. Open Science Framework DoI:10.31219/osf.io/jw9f6, arXiv: 1104.0032. First publ. 2011.

Puntigam, R. A., Lämmerzahl, C., Hehl, F. W. (1997): Maxwell's theory on a post-Riemannian spacetime and the equivalence principle. Class. Quantum Grav. $\mathbf{1 4}^{5}, 1347-1356$. DOI:10 10 88/0264-9381/14/5/033.

Rindler, W. (1986): Essential Relativity: Special, General, and Cosmological, rev. 2nd ed. (Springer, New York). First publ. 1969. Dor:10. 1007/978- 1-4757-1135- 6.

Röhr, N., Uggla, C. (2005): Conformal regularization of Einstein's field equations. Class. Quantum Grav. 22 ${ }^{17}$, 3775-3787. Dor:10. 1088/0264-9381/22/17/026.

Schouten, J. A. (1954): Ricci-Calculus: An Introduction to Tensor Analysis and Its Geometrical Applications, 2nd ed. (Springer, Berlin). First publ. in German 1924. Dor:10. 1007/978 - 3- 662 - 12927 - 2 .

- (1989): Tensor Analysis for Physicists, corr. 2nd ed. (Dover, New York). First publ. 1951.

Segev, R. (2000): Notes on stresses for manifolds. Rend. Sem. Mat. Univ. Pol. Torino 58 ${ }^{2}$, 199-206. https://www. emis. de/journals/RSMT/58-2. html.

- (2001): A correction of an inconsistency in my paper "Cauchy's theorem on manifolds". J. Elast. 63 ${ }^{1}$, 55-59. See Segev, Rodnay (1999). DOI:10. 1023/A : 1013085312562.

- (2002): Metric-independent analysis of the stress-energy tensor. J. Math. Phys. $43^{6}, 3220$ 3231. http://www. bgu.ac.il/ rsegev/Papers/MetricIndependent.pdf, DOI:10. 106 3/1.1475347.

Segev, R., Rodnay, G. (1999): Cauchy's theorem on manifolds. J. Elast. 56 ${ }^{2}, 129-144$. See also erratum Segev (2001). DOI:10. 1023/A: 1007651917362. 
Smarr, L., Taubes, C., Wilson, J. R. (1980): General relativistic hydrodynamics: the comoving, Eulerian, and velocity potential formalisms. In: Tipler (1980): ch. 11:157-183. DoI:10.1016 /B978- 0-12-691380-4.50017- 4.

Smarr, L., York Jr., J. W. (1978): Kinematical conditions in the construction of spacetime. Phys. Rev. D 17 ${ }^{10}$, 2529-2551. Dor:10.1103/PhysRevD. 17. 2529.

Smarr, L. L., ed. (1979): Sources of Gravitational Radiation. (Cambridge University Press, Cambridge).

Synge, J. L. (1960a): Relativity: The General Theory. (North-Holland, Amsterdam).

— (1960b): Classical Dynamics. In: Flügge (1960): I-VII, 1-225, 859-902. DOI:10 . 1007/978 - 3- 642 - 45943- 6 - 1.

Tipler, F. J., ed. (1980): Essays in General Relativity: A Festschrift for Abraham Taub. (Academic Press, New York). DOI:10.1016/C2013-0-11601- 7.

Tolman, R. C. (1949): Relativity, Thermodynamics and Cosmology. (Oxford University Press). First publ. 1934.

Truesdell III, C. A., Toupin, R. A. (1960): The Classical Field Theories. In: Flügge (1960): I-VII, 226-902. With an appendix on invariants by Jerald LaVerne Ericksen. DOI:10 . 1007/97 8-3-642-45943-6_2.

Vallis, G. K. (2006): Atmospheric and Oceanic Fluid Dynamics: Fundamentals and Large-scale Circulation. (Cambridge University Press, Cambridge). DOI:/10 . 1017/9781107588417.

Wilson, J. R., Mathews, G. J. (2007): Relativistic Numerical Hydrodynamics, repr. (Cambridge University Press, Cambridge). First publ. 2003. Dor:10. 1017/CB09780511615917.

York Jr., J. W. (1979): Kinematics and dynamics of general relativity. In: Smarr (1979): 83-126. 